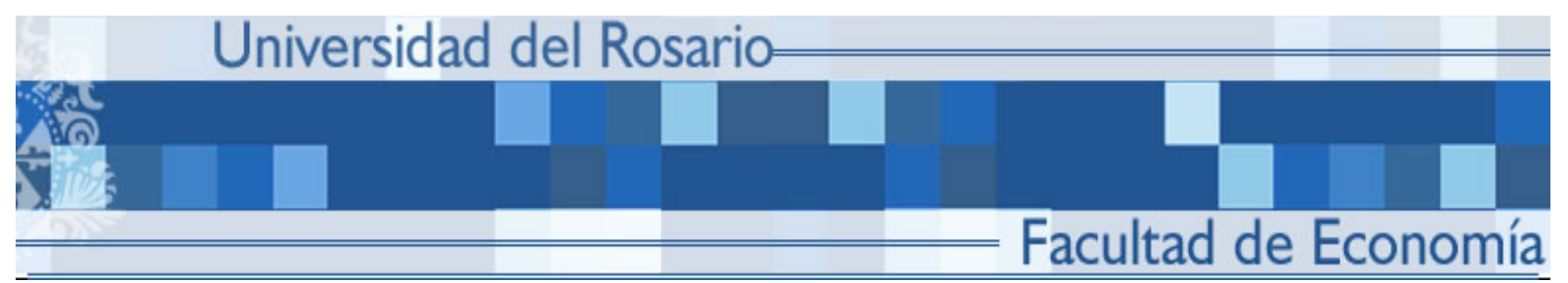

\title{
HETEROGENEITY AND THE FORMATION OF RISK-SHARING COALITIONS
}

Fernando Jaramillo

Hubert Kempf

Fabien Moizeau

\section{SERIE DOCUMENIOS DE TRABAJ O}

No. 143

Septiembre de 2013 


\title{
Heterogeneity and the Formation of Risk-Sharing Coalitions.*
}

\author{
Fernando JARAMILLO† Hubert KEMPF ${ }^{\ddagger}$ and Fabien MOIZEAU§ \\ Revised version
}

February 2013.

\begin{abstract}
We offer a new explanation of partial risk sharing based on coalition formation and segmentation of society in a risky environment, without assuming limited commitment and imperfect information. Heterogenous individuals in a society freely choose with whom they will share risk. A partition belonging to the core of the membership game obtains. Perfect risk sharing does not necessarily arise. Focusing on mutual insurance rule and assuming that individuals only differ with respect to risk, we show that the core partition is homophily-based. The distribution of risk affects the number and size of these coalitions. Individuals may pay a lower risk premium in riskier societies. A higher heterogeneity in risk leads to a lower degree of risk sharing. We discuss how the endogenous partition of society into risk-sharing coalitions may shed light on empirical evidence on partial risk sharing. The case of heterogenous risk aversion leads to similar results.
\end{abstract}

Keywords: Risk Sharing, Group Membership, Social Segmentation.

JEL Classification: C71, D3, D71, D81.

\footnotetext{
*We are very grateful to David Bardey, Max Engers, Laszlo Koczy, Sophie Larribeau, Thierry Magnac as well as to conference participants at Coalition Theory Network Workshop 2009, the PET 09 Galway, the Journées Louis-André Gérard-Varet \#9 and the EEA Glasgow meeting 2010, and seminar participants at Université de Lille 1, Tinbergen Institute, Banque de France, Université du Maine, Université de Strasbourg, Université de Clermont 1, Université de Montpellier 1, SMART (INRA, Rennes), Université de Cergy-Pontoise and Universidad del Rosario (Bogota).

${ }^{\dagger}$ Universidad del Rosario, Bogota (Colombia), email: fernando.jaramillo@urosario.edu.co.

${ }^{\ddagger}$ Ecole Normale Supérieure de Cachan and Paris School of Economics, email: hubert.kempf@ens-cachan.fr.

${ }^{\S}$ CREM (Condorcet Center), Université de Rennes 1 and Institut Universitaire de France, email: fabien.moizeau@univ-rennes1.fr.
} 


\section{Introduction.}

In developing economies where financial markets are lacking, individual incomes vary widely (see, in particular, Townsend, 1994, for ICRISAT villages in India or Dubois, Jullien and Magnac, 2008, for Pakistan villages). Nonetheless, the idiosyncratic part of income risk is relatively large, suggesting that insurance against shocks is desirable (Townsend, 1995, Dercon, 2004). Thus we should expect risk-averse households to pool risk in order to smooth consumption. If risk is fully insured, theory tells us that individual consumption is determined by aggregate consumption (see Borch, 1962, Arrow, 1964, Wilson, 1968). However, this proposition has been subject to many empirical rebuttals. In developing economies, the empirical evidence supports partial risk sharing. Households are able to protect consumption against adverse income shocks but full insurance is not achieved (see, among many others, Townsend, 1994, Kazianga and Udry, 2005). ${ }^{1}$ Moreover, empirical works identify risksharing groups and networks smaller than the entire society. For instance, Fafchamps and Lund (2003) show that mutual insurance is implemented within confined networks of families and friends. Mazzocco and Saini (2010), using ICRISAT data, show that the relevant unit to test for efficient risk sharing is the caste and not the village. Other individual characteristics also appear to be key determinants of membership in risk-sharing groups or networks. Geographic proximity as well as age and wealth differences also play a role in the formation of networks (see Fafchamps and Gubert, 2007). Using data on group-based funeral insurance in Ethiopia and Tanzania, Dercon et alii (2006) provide evidence of assortative matching according to physical distance, kinship, household size and age. Arcand and Fafchamps (2012) find robust evidence of individuals' sorting with respect to physical or ethnic proximity as well as wealth and household size for communitybased organizations in Senegal and Burkina Faso. ${ }^{2}$ It turns out that the distribution of individuals' attributes over the population plays a key role in group memberships and the extent of risk sharing.

Two explanations of partial risk sharing in developing economics can be found in the theoretical literature: imperfect information ${ }^{3}$ and limited commitment ${ }^{4}$. Here, we offer a third explanation based on the capacity of agents to voluntarily form risk-sharing groups. This generates a seg-

\footnotetext{
${ }^{1}$ Townsend (1995), Ray (1998), Dubois (2002), or Dercon (2004) are excellent surveys of the literature. For developped economies also, empirical evidence does not support the full insurance hypothesis (see Mace, 1991, Cochrane, 1991, Hayashi, Altonji and Kotlikoff, 1996, Attanasio and Davis, 1996).

${ }^{2}$ See also the survey of Fafchamps (2008) on the role of families and kinship networks in sharing risk.

${ }^{3}$ See Atkeson and Lucas (1992) and Ligon (1998) for an explanation of inefficient risk sharing based on moral hazard. See also Deb and Suri (2012) who develop a model of informal credit through farming contract under hold-up problem, moral hazard and limited liability.

${ }^{4}$ See Kocherlakota (1996), Ligon, Thomas and Worrall (2002) for studies of efficient dynamic contracts in the absence of commitment. See Genicot and Ray (2003) and Bold (2009) who investigate self-enforcing risk-sharing agreements which are robust to coalitional deviations.
} 
mentation of society into a plurality of distinct groups formally defined by a core partition. Our analysis allows us to understand how ex ante heterogeneity among individuals shapes the pattern of risk-sharing coalitions and thus limits the extent of risk sharing. Our approach relies both on commitment and perfect information on the individual's characteristics.

Formally, we first study a society comprised of many individuals, each one characterized by the three following attributes : risk aversion, endowment and risk, defined as the variance of the distribution from which is drawn an idiosyncratic shock. Individuals are characterized by a CARA utility function; they may form a group in order to share risk. Individuals commit to sharing their resources Pareto-optimally with members of their risk-sharing group. No specific risk-sharing rule is a priori assumed. We examine the segmentation of society into such risk-sharing groups. ${ }^{5}$ We provide sufficient conditions on Pareto-weights attached on individuals in the social welfare function for existence of a core partition. We show that for an optimal risk-sharing rule and a distribution of individuals' attributes it is likely that the society is partitioned into distinct coalitions. Hence, even when commitment to a Pareto-optimal allocation of resources and perfect information on individuals' characteristics are assumed, perfect risk sharing does not necessarily arise when heterogenous agents freely choose their risk-sharing group.

In order to draw sharper conclusions on the link between ex ante heterogeneity among individuals and risk sharing we consider a specific risk-sharing rule: individuals share the random component of their income equally with members of their risk-sharing group. We refer to this rule as the mutual insurance rule. Individuals only differ with respect to their risk. ${ }^{6}$ We show that the resulting core partition is unique (under some mild assumption). It turns out that the key variable determining the coalition formation process is relative risk heterogeneity, as measured by the variance ratios between individuals. This leads the core partition to be homophily-based: coalitions pool agents similar with respect to the variance of the idiosyncratic shock. In this perspective, the extent of risk sharing is limited by the formation of coalitions due to heterogeneity. Two individuals belonging to the same society do not necessarily share risk in the same coalition.

We study the impact of specific variance schedules on the core partition and show thanks to these cases how the number and the size of coalitions belonging to the core partition are affected by the distribution of risk within society.

Defining an aggregate risk premium index and analyzing two societies with an equal number of individuals, we compare the amount of resources devoted to risk sharing. We prove that a more

\footnotetext{
${ }^{5}$ Ghatak (1999), Chiappori and Reny (2005), Genicot (2006), Legros and Newman (2007), study risk-sharing groups formation but these works consider that the size of groups is exogenously given.

${ }^{6}$ This could be justified by the fact that individuals do not use the same technology and are differently exposed to risks (see for instance Conley and Udry, 2010).
} 
risky society (in the sense of second-order stochastic dominance) may devote less resources to risk sharing than a less risky one. This implies that some individuals may prefer to live in the more risky society. This result too hinges on relative risk heterogeneity.

Further, we proceed to some comparative statics concerning the size of population. We show that replication of society does not modify the boundaries of risk-sharing coalitions of the core partition. On the opposite, the core partition may be modified when individuals characterized by new risks are added to society. It turns out that none of these ways of increasing the size of society, even though allowing for risk diversification, actually lead to the grand coalition and perfect risk sharing.

We discuss the empirical implications of our model. Most empirical studies have found evidence of partial risk sharing. This can be explained by the fact that individuals sort themselves into risksharing groups smaller than the whole society. We show that the coefficients of the consumption function's specification used in econometric studies depend on the number and size of risk-sharing coalitions. Following empirical works (see in particular, Jalan and Ravallion, 1999, and Suri, 2009), we consider that the average value of the coefficient on individual income measures the extent of risk sharing. One implication is that this coefficient is larger, respectively smaller, when there is more, respectively less, discrepancy between idiosyncratic shocks variances. Given our theoretical prediction on the key role played by relative risk heterogeneity on the extent of risk sharing, we claim that variables such as risk-ratios should be introduced in dyadic regressions used for assessing the probability of forming a link between two individuals (Fafchamps and Gubert, 2007 and Arcand and Fafchamps, 2012, use dyadic analysis to assess risk sharing).

These results highlight that these two dimensions (extent of risk sharing and aggregate risk premium) matter for the assessment of risk sharing in society when social segmentation endogenously emerges. Take two societies, one more risky than the other. The more risky society may be characterized by a higher aggregate risk premium. According to this dimension, we may conclude that insurance against risk is worse in this society. However, if the more risky society is more homogenous leading to larger risk-sharing coalitions, then the extent of risk sharing would be larger which is commonly interpreted as a better insurance outcome.

In order to check the robustness of our approach, we discuss the alternative case where individuals differ in their aversion to risk. That is, we focus on heterogeneous individual utility functions. We prove that our propositions carry over to this case, even though the logic behind the formation of the core partition differs. In this case, all agents are willing to pool with the most risk tolerant agents.

The relationship between risk and group formation has already been studied by various authors. 
In particular, Genicot and Ray (2003) develop a group formation approach where one risk-sharing coalition must be robust to potential subgroup deviations. This stability condition may limit the size of the risk-sharing coalition. Bold (2009) solves for the optimal dynamic risk-sharing contract in the set of coalition-proof equilibria. We depart from these works in two ways. First, we focus on heterogeneity of individuals' attributes as the force limiting the size of risk-sharing coalition instead of the absence of commitment. Second, we study the partition of society into possibly multiple coalitions. Taub and Chade (2002) study under which conditions a core partition is immune to future individual defections. Our focus is different as we build a setup that allows us to characterize a relationship between (i) the risk characteristics of a society, (ii) the membership and size of risksharing groups and (iii) the extent of risk coping. Our paper bears some similarities with Henriet and Rochet (1987) who develop a model of endogenous formation of mutuals using a cooperative game theoretical approach. The modelling strategy is different from ours as they assume a continuum of agents, the existence of congestion costs and a binomial distribution of shock. Further, they focus on formal insurance activity and do not address the issue of mutualization of risk under informal insurance schemes. Finally, Bramoullé and Kranton (2007) develop a model of network formation to tackle the risk sharing issue. As they consider identical individuals, they do not examine how heterogeneity shapes the architecture of networks.

The plan of the paper is as follows. In the following section, we present our coalition-formation framework with individuals differing with respect to the exposure to risk. We then characterize the partition that emerges and study the relationship between the risk distribution, the size of risksharing groups and the extent of risk sharing. Section 4 discusses the empirical implications of our theoretical setup. In section 5, we study a coalition-formation framework where individuals differ with respect to their risk aversion. Section 6 concludes. $^{7}$

\section{The Model.}

We consider a society $\mathbf{I}$ formed of $N$ agents, indexed by $i=1, \ldots, N$. These individuals live $T$ periods. There is no production in this society and agents are endowed with quantities of a non-storable good. At each date $t$, the endowment $y_{i t}$ allotted to individual $i$ has a deterministic component $w_{i t}$ and is affected by an idiosyncratic risk $\varepsilon_{i t}$ and a common shock $\nu_{t}$ :

$$
y_{i t}=w_{i t}+\varepsilon_{i t}+\nu_{t}
$$

where $\nu_{t}$ is i.i.d across individuals and time and normally distributed: $\nu_{t} \rightsquigarrow \mathcal{N}\left(0, \sigma_{\nu}^{2}\right)$. Moreover, $\varepsilon_{i t}$ is i.i.d. across time and normally distributed: $\varepsilon_{i t} \rightsquigarrow \mathcal{N}\left(0, \sigma_{i}^{2}\right)$. Let the state of nature at each date

\footnotetext{
${ }^{7}$ Proofs of Propositions and Lemmas are provided in the Appendix.
} 
$t$ be denoted by $\epsilon_{t}=\left(\nu_{t}, \varepsilon_{1 t}, \ldots, \varepsilon_{j t}, \ldots \varepsilon_{N t}\right)$.

Individuals have instantaneous CARA utility functions and, at date 0 , agent $i$ is characterized by the following expected utility function:

$$
U_{i}=-\mathbb{E}_{0}\left[\frac{1}{\alpha_{i}} \sum_{t=1}^{T} \delta^{t-1} e^{-\alpha_{i} c_{i t}\left(\epsilon_{t}\right)}\right]
$$

with $\mathbb{E}_{0}$ the mathematical expectation operator at date $0, \alpha_{i}$ the absolute risk aversion parameter, $\delta$ the discount factor and $c_{i t}$ the consumption of agent $i$ at date $t$ in state $\epsilon_{t}$.

Hence, we denote by $\Gamma_{i}$ the vector of individual $i$ 's characteristics:

$$
\Gamma_{i}=\left(\sigma_{i}, w_{i}, \alpha_{i}\right)
$$

There is perfect information in the following sense: the various idiosyncratic variances are public information and the realizations of shocks are also perfectly observed by all agents when they occur. It is assumed that there are no financial markets allowing any agent to insure himself against his idiosyncratic risks. But agents have the possibility to form groups in order to cope with risk according to a given transfer scheme.

\subsection{Risk-sharing Coalitions.}

Individuals have the possibility to group themselves in order to pool risk. A group is called a risk-sharing coalition or club of individuals and a partition of the society is a set of risk-sharing coalitions. More formally, we use the following

Definition 1 A non-empty subset $S_{j}$ of $\mathbf{I}$ is called a risk-sharing coalition and $\mathcal{P}=\left\{S_{1}, \ldots, S_{j}, \ldots, S_{J}\right\}$ for $j=1, \ldots, J$ is called a partition of $\mathbf{I}$ if (i) $\bigcup_{j=1}^{J} S_{j}=\mathbf{I}$ and (ii) $S_{j} \cap S_{j^{\prime}}=\emptyset$ for $j \neq j^{\prime}$.

According to this definition, any individual belongs to one and only one risk-sharing coalition. The size of the $j-t h$ coalition, $S_{j} \subseteq \mathbf{I}$, is denoted by $n_{j}$.

We make two key assumptions. First, once risk-sharing coalitions are formed, we assume commitment in the allocation of resources. This is a key difference with, for instance, Genicot and Ray (2003) or Bold (2009). This commitment assumption allows us to make more transparent the link between ex ante heterogeneity and the extent of risk-sharing in the society. Second, we require that the coalition's resources be Pareto-optimally allocated between members of the coalition and we do not assume a priori any risk-sharing rule, that is any transfer scheme. For any coalition $S$ $\subseteq$ I, Pareto-optimal allocations of resources between members of $S$ are solutions of the following program

$$
\max _{\left\{c_{i t}\left(\epsilon_{t}\right)\right\}} \sum_{i \in S} \mu_{i}(S)\left(-\mathbb{E}_{0}\left[\frac{1}{\alpha_{i}} \sum_{t=1}^{T} \delta^{t-1} e^{-\alpha_{i} c_{i t}\left(\epsilon_{t}\right)}\right]\right)
$$


subject to the following feasibility constraint at each date $t$ and each state $\epsilon_{t}$ :

$$
\sum_{i \in S} c_{i t}\left(\epsilon_{t}\right) \leq \sum_{i \in S} y_{i t}\left(\epsilon_{t}\right)
$$

where $\mu_{i}(S)$ denotes the non-negative Pareto-weight attached on individual $i$ belonging to $S$.

It turns out that Pareto-optimal consumptions, denoted by $c_{i}(S)$, are equal to: ${ }^{8}$

$$
c_{i}(S)=\frac{1}{\alpha_{i}}\left[\ln \mu_{i}(S)-\frac{\sum_{k \in S} \frac{\ln \mu_{k}(S)}{\alpha_{k}}}{\sum_{k \in S} \frac{1}{\alpha_{k}}}\right]+\frac{\frac{1}{\alpha_{i}}}{\sum_{k \in S} \frac{1}{\alpha_{k}}} \sum_{k \in S}\left(w_{k}+\varepsilon_{k}+\nu\right), \forall i \in S .
$$

For each agent $i \in S$ and coalition $S \subseteq \mathbf{I}, c_{i}(S)$ characterizes the set of allocations entailed by optimal risk-sharing rules, each one being associated to a particular value of $\mu_{i}(S)$. At this stage, in order to be as general as possible, we allow Pareto-weights to depend on the membership of $S$.

For any $S \subseteq \mathbf{I}$, let us denote by $|S|$ the cardinal of $S$ and $\Gamma_{S}$ the characteristics of $S$, that is

$$
\Gamma_{S} \equiv\left\{\left(\Gamma_{i}\right)_{i \in S}\right\}
$$

Optimal consumption of individual $i$ in coalition $S$ depends on $\Gamma_{S}$ as it is expressed by the characteristics of all members of coalition $S$ and their assigned weight $\mu_{k}(S)$ in $S$. We will prove that heterogeneity between individuals plays a key role in the choice for each agent of their membership.

\subsection{The Core Partition.}

To address the issue of segmentation of society into risk-sharing coalitions, we consider the following sequence of events:

1. Agents form risk-sharing coalitions and a partition of society is obtained.

2. Individuals commit to applying a Pareto-optimal allocation of resources within the coalition they belong to.

3. Idiosyncratic shocks are realized. Agents then consume their optimal level of resources.

We solve this coalition-formation game by looking at a core partition defined as follows:

Definition 2 A partition $\mathcal{P}^{*}=\left\{S_{1}^{*}, \ldots, S_{j}^{*}, \ldots, S_{J}^{*}\right\}$ belongs to the core of the coalition-formation game if:

$$
\nexists £ \subseteq \mathbf{I} \text { such that } \forall i \in £, V_{i}(£)>V_{i}\left(\mathcal{P}^{*}\right)
$$

where $V_{i}\left(\mathcal{P}^{*}\right)$ denotes the utility for agent $i$ associated with partition $\mathcal{P}^{*}$.

\footnotetext{
${ }^{8}$ See for instance Wilson (1968). If we considered a production sector and leisure choice, formulas of Pareto-optimal consumptions would not be affected if separable utility functions are assumed (see Townsend, 1994). The good being assumed non-storable, the time subscript is dropped in the sequel.
} 
According to this definition, a core partition is such that there is no blocking coalition, that is there is no profitable coalitional deviation.

We focus on two alternative assumptions about Pareto-weights and prove that a core partition exists when the social welfare function relies on one or the other:

Proposition 1 A core partition exists if Pareto-weights satisfy either

(i) Assumption 1

$$
\ln \mu_{i}(S)=\Psi\left(\Gamma_{i}\right)+\Omega\left(\Gamma_{S}\right), \text { with } \Psi: \mathbb{R}_{+}^{3} \rightarrow \mathbb{R} \text { and } \Omega: \mathbb{R}_{+}^{3|S|} \rightarrow \mathbb{R}, \forall i=1, \ldots, N, \forall S \subseteq \mathbf{I}
$$

or

(ii) Assumption 2

$$
\ln \mu_{i}(S)=\alpha_{i}\left(w_{i}-\frac{1}{2} \frac{\sigma_{i}^{2}+\sigma_{\nu}^{2}}{\sum_{m \in S} \frac{1}{\alpha_{m}}}\right), \forall i=1, \ldots, N, \forall S \subseteq \mathbf{I} .
$$

Proposition 1 provides two characterizations of Pareto-weights that satisfy the common ranking property. It is known to be a sufficient condition for the non-emptiness of the core partition (see Farrell and Scotchmer, 1988, and Banerjee, Konishi and Sönmez, 2001). The common ranking property implies that all individuals agree on the ranking of coalitions to which they belong.

Under Assumptions 1 and 2, benchmark cases of risk-sharing rules can be analyzed.

First, Assumption 1 allows us to us consider the utilitarian social welfare function, i.e. $\ln \mu_{i}(S)=$ $\ln \mu$ for any $i \in \mathbf{I}$ which satisfies Assumption 1. It turns out that (1) can be expressed as follows

$$
c_{i}(S)=\frac{\frac{1}{\alpha_{i}}}{\sum_{k \in S} \frac{1}{\alpha_{k}}} \sum_{k \in S}\left(w_{k}+\varepsilon_{k}+\nu\right), \forall i \in S
$$

leading that any individual $i$ consumes a fraction of the coalition resources, this fraction being equal to the ratio of his risk tolerance, i.e. $1 / \alpha_{i}$, to the coalition $S$ risk tolerance, i.e. $\sum_{k \in S}\left(1 / \alpha_{k}\right)$. If individuals have the same risk aversion, this leads to the equal risk-sharing rule. ${ }^{9}$

Second, Assumption 1 covers the case where $\frac{\ln \mu_{i}(S)}{\alpha_{i}}=w_{i}-\frac{\alpha_{i}}{2} \sigma_{i}^{2}$ for any $i \in \mathbf{I}$. For these definitions of Pareto-weights, the following obtains

$$
c_{i}(S)=w_{i}-\frac{\alpha_{i}}{2} \sigma_{i}^{2}+\frac{\frac{1}{\alpha_{i}}}{2 \sum_{k \in S} \frac{1}{\alpha_{k}}} \sum_{k \in S}\left(\alpha_{k} \sigma_{k}^{2}\right)+\frac{\frac{1}{\alpha_{i}}}{\sum_{k \in S} \frac{1}{\alpha_{k}}} \sum_{k \in S}\left(\varepsilon_{k}+\nu\right), \forall i \in S .
$$

In the Proof of Proposition 1, we show that bargaining between members of coalition $S$ generates a Nash solution which is equal to these optimal levels of consumption.

\footnotetext{
${ }^{9}$ The implications of equal risk sharing on the formation of risk-sharing networks have been studied theoretically (see Bramoullé and Kranton, 2007) and experimentally (see Barr et alii., 2011).
} 
Third, we can also consider under Assumption 1 that if $\frac{\ln \mu_{i}(S)}{\alpha_{i}}=w_{i}$ for any $i \in \mathbf{I}$ then consumption levels can be expressed as

$$
c_{i}(S)=w_{i}+\frac{\frac{1}{\alpha_{i}}}{\sum_{k \in S} \frac{1}{\alpha_{k}}} \sum_{k \in S}\left(\varepsilon_{k}+\nu\right), \forall i \in S .
$$

This risk-sharing rule amounts to share the whole stochastic resources of coalition $S$. Each individual obtains a fraction of this amount defined as the ratio of his risk tolerance to the coalition $S$ risk tolerance. Calling it the mutual insurance rule, we study its implications in the next section (see also Ray, 1998, for a study of such a rule).

Fourth, under Assumption 2, optimal consumption (1) is expressed as follows

$$
c_{i}(S)=w_{i}-\frac{1}{2 \sum_{m \in S} \frac{1}{\alpha_{m}}}\left(\sigma_{i}^{2}+\sigma_{\nu}^{2}-\frac{\frac{1}{\alpha_{i}}}{\sum_{k \in S} \frac{1}{\alpha_{k}}} \sum_{k \in S}\left(\sigma_{k}^{2}+\sigma_{\nu}^{2}\right)\right)+\frac{\frac{1}{\alpha_{i}}}{\sum_{k \in S} \frac{1}{\alpha_{k}}} \sum_{k \in S}\left(\varepsilon_{k}+\nu\right), \forall i \in S .
$$

This rule has been studied by Baton-Lemaire (1981) and has the noteworthy implication that when individuals have the same risk aversion, it leads the grand coalition to be in the core of the coalition formation game whatever heterogeneity in $\mathbf{I}$, that is, whatever the distributions of $\sigma_{i}^{2}$ and $w_{i}$ (see Proof of Proposition 1).

We can then offer the following

Proposition 2 The grand coalition is not always in the core.

For an optimal risk-sharing rule and a set of individual characteristics $\Gamma_{\mathbf{I}}$, it is likely that the society is partitioned into distinct coalitions. Given that individuals commit to sharing Paretooptimally the coalition's resources, perfect risk sharing obtains when the grand coalition belongs to the core. This Proposition amounts to say that perfect information and commitment to a Paretooptimal allocation of resources do not inevitably imply perfect risk sharing when heterogenous agents freely choose their risk-sharing group. These individual decisions generate the formation of a plurality of risk-sharing coalitions reflecting the heterogeneity of agents individuals. In other words, the endogenous formation of risk-sharing coalitions possibly leads to partial risk sharing. The rest of the paper is devoted to understanding the relationship between heterogeneity and partial risk sharing.

\section{A Particular Optimal Risk-Sharing Rule: Mutual Insurance.}

We consider a particular optimal risk-sharing rule which is the mutual insurance rule and assume that individuals have the same risk aversion parameter. As said before, for this rule consumption 
levels are given by (2) and can be rewritten as:

$$
c_{i}(S)=w_{i}+\frac{\sum_{k \in S} \varepsilon_{k}}{n}+\nu .
$$

When the non-stochastic component is identical for all agents, this rule amounts to the equal sharing rule. This rule has the crucial advantage of focusing on transfers among agents solely justified by the objective of sharing risk among individuals as these transfers relate to the random components of income. In other words, we abstract from any redistribution motive not related to risk sharing. All our results will be deduced from this sole rationale.

The expected utility of individual $i$ in group $S, V_{i}(S)$, is:

$$
V_{i}(S)=-\mathbb{E}\left[\frac{1}{\alpha} e^{-\alpha w_{i}-\alpha \frac{\sum_{k \in S} \varepsilon_{k}}{n}-\alpha \nu}\right]
$$

As we assume a CARA utility function and normal distribution for each idiosyncratic shock, the Arrow-Pratt approximation is exact:

$$
V_{i}(S)=-\frac{1}{\alpha} e^{-\alpha\left[w_{i}-\frac{\alpha}{2 n^{2}} \sum_{k \in S} \sigma_{k}^{2}-\frac{\alpha}{2} \sigma_{\nu}^{2}\right]} .
$$

We define the certainty-equivalent income for individual $i$ in group $S$, denoted by $\omega_{i}(S)$, as:

$$
\omega_{i}(S)=w_{i}-\frac{\alpha}{2} \sum_{k \in S} \frac{\sigma_{k}^{2}}{n^{2}}-\frac{\alpha}{2} \sigma_{\nu}^{2} .
$$

The risk premium for any individual $i$ in group $S$, denoted by $\pi(S)$, is equal to $\frac{\alpha}{2} \sum_{k \in S} \frac{\sigma_{k}^{2}}{n^{2}}+\frac{\alpha}{2} \sigma_{\nu}^{2}$ and thus is the same for every member of $S$.

The individual gain for agent $i$ from membership to group $S$ rather than to group $S^{\prime}$ amounts to a reduction in risk premium:

$$
\pi\left(S^{\prime}\right)-\pi(S)
$$

In other words, an agent prefers joining a group (provided she is accepted in this group) in which her certainty-equivalent income is higher. The more risky an agent, the more he benefits from belonging to a given group (rather than remaining alone): individual gains from a group are differentiated and actually increasing with the riskiness of the agent. This is the core characteristics of a group functioning under our insurance rule. Hence, the formation of a group relies on the trade-off between heterogeneity and size. Accepting a new member has two opposite effects: on the one hand, everything else equal, the higher its size, the lower the risk premium; on the other hand, accepting an individual increases the sum of individual risks leading members to pay a higher risk premium. Therefore when assessing the net benefit of accepting a given individual, characterized by a particular variance, an insider has to weigh these two effects. 
We have the following set of individual risk aversions

$$
\vec{\sigma}_{I}=\left\{\sigma_{1}^{2}, \sigma_{2}^{2}, \ldots \sigma_{N}^{2}\right\}
$$

and without loss of generality, we index individuals as follows: for $i$ and $i^{\prime}=1, . ., N$ with $i<i^{\prime}$ then $\sigma_{i}^{2}<\sigma_{i^{\prime}}^{2}$. We will thus say that a lower indexed individual is a"less risky agent"(strictly speaking, individual risk is associated with the law of motion of $\varepsilon_{i}$ ).

Given these differences among individuals, we define $\lambda_{i} \equiv \frac{\sigma_{i}^{2}}{\sigma_{i-1}^{2}}$ for $i=2, \ldots, N . \lambda_{i}$ is called the"risk ratio" between agents $i-1$ and $i$. We will use the following

Definition 3 Any society $\mathbf{I}$ can be characterized by a risk-ratio schedule $\Lambda=\left\{\lambda_{2}, \lambda_{3}, \ldots, \lambda_{N}\right\}$ for $i=2, \ldots, N$.

This risk-ratio schedule captures the relative risk heterogeneity within society.

\subsection{The Characteristics of the Core Partition.}

In this section, we provide results on the impact of individual heterogeneity with respect to risk on the segmentation of society in multiple risk-sharing coalitions.

From Proposition 1, we know that under a mutual insurance rule the core is not empty. We are then able to offer the following:

Proposition 3 A core partition $\mathcal{P}^{*}=\left\{S_{1}^{*}, \ldots, S_{j}^{*}, \ldots, S_{J}^{*}\right\}$ is characterized as follows:

i It is unique if

$$
\forall z=2, \ldots, N-1, \quad \frac{\lambda_{z+1}-\lambda_{z}}{\lambda_{z+1}-1} \geq-\frac{1}{z+1}
$$

ii / It is consecutive, that is, if $i$ and $\widetilde{i}$ both belong to $S_{j}^{*}$ then $\forall i^{\prime}, i>i^{\prime}>\widetilde{i}, i^{\prime} \in S_{j}^{*}$.

iii/ For any two individuals $i \in S_{j}^{*}$ and $i^{\prime} \in S_{j^{\prime}}^{*}$ such that $\sigma_{i}^{2}<\sigma_{i^{\prime}}^{2}$, then $\pi\left(S_{j}^{*}\right) \leqq \pi\left(S_{j^{\prime}}^{*}\right)$.

The first result, $i /$, provides a sufficient condition for the core partition to be unique. The condition on uniqueness depends on the rank of individuals. If the risk ratios are increasing with the index $z$, this condition is always met. The condition may appear stringent when $\lambda_{z}>\lambda_{z+1}, \forall z=$ $2, \ldots, N-1$. The expression $\frac{-1}{z+1}$ is an increasing function of $z$ which equals $\frac{-1}{3}$ when $z=2, \frac{-1}{N}$ when $z=N-1$, and tending to 0 when $N$ is sufficiently large. ${ }^{10}$

\footnotetext{
${ }^{10}$ Let us stress that the core partition is generically unique (see for instance Farrell and Scotchmer, 1988) but we need to provide a sufficient condition for uniqueness in order to proceed to our comparative static analysis.
} 
Turning to the characteristics of the core partition, the second result, ii/, is about consecutivity which captures the homophily feature. Coalitions belonging to the core partition include agents who are "close" in terms of exposure to risk. Take an individual who has to choose between two individuals in order to form a risk-sharing coalition. It is easy to check that he always prefers the less risky of the pair. This implies that if an agent $i$ is willing to form a coalition with some other agent $i^{\prime}$, then all agents with a lower risk than $i^{\prime}$ are also accepted by $i$ in the coalition. ${ }^{11}$

The third result, iii/, is in line with consecutivity. Take the less risky individual characterized by $\sigma_{1}^{2}$. He is accepted by any possible coalition and chooses the group that incurs the lowest risk premium. More risky individuals may not be accepted by agents characterized by low risks to pool resources in a same group. They thus pay a higher risk premium in other coalitions. ${ }^{12}$

Given the consecutivity property, from now on, we adopt the following convention that for any $S_{j}^{*}$ and $S_{j^{\prime}}^{*}, j^{\prime}>j$ when $\sigma_{i}^{2}<\sigma_{i^{\prime}}^{2}, \forall i \in S_{j}^{*}, \forall i^{\prime} \in S_{j^{\prime}}^{*}$. Another way to express consecutivity is to say that a core partition can be characterized by a series of "pivotal agents", that is agents who are the most risky agents of the coalition they belong to:

Definition 4 Given the coalition $S_{j}^{*}$ of size $n_{j}$ in the core-partition, the pivotal agent, defined by the integer $p_{j} \in\{1, \ldots, N\}$, associated with $S_{j}^{*}$ and the next agent $p_{j}+1$ are characterized by variances $\sigma_{p_{j}}^{2}$ and $\sigma_{p_{j}+1}^{2}$, respectively, such that:

$$
\pi\left(S_{j}^{*} \backslash\left\{p_{j}\right\}\right) \geq \pi\left(S_{j}^{*}\right) \text { and } \pi\left(S_{j}^{*} \cup\left\{p_{j}+1\right\}\right)>\pi\left(S_{j}^{*}\right)
$$

Hence,

$$
\sigma_{p_{j}}^{2} \leq\left[2 n_{j}-1\right] \sum_{k \in S_{j}^{*} \backslash\left\{p_{j}\right\}} \frac{\sigma_{k}^{2}}{\left(n_{j}-1\right)^{2}}
$$

and

$$
\sigma_{p_{j}+1}^{2}>\left[2 n_{j}+1\right] \sum_{k \in S_{j}^{*}} \frac{\sigma_{k}^{2}}{n_{j}^{2}} .
$$

\footnotetext{
${ }^{11}$ The consecutivity property is obtained in other models of risk-sharing agreements (see for instance Henriet and Rochet, 1987, and Legros and Newman, 2007).

${ }^{12}$ Let us remark that the CARA specification is not crucial for the results obtained. If we assume an increasing and concave utility function $u(c)$ and infinitesimal shocks, then using the Arrow-Pratt approximation would yield the following risk premium for any individual $i$ in group $S$

$$
\pi_{i}(S)=-\frac{u^{\prime \prime}\left(w_{i}\right)}{u^{\prime}\left(w_{i}\right)} \frac{\sum_{k \in S} \sigma_{k}^{2}}{|S|^{2}} .
$$

Hence, the purpose for each individual remains to obtain the lowest ratio $\frac{\sum_{k \in S} \sigma_{k}^{2}}{|S|^{2}}$. However, when $u($.$) is not CARA$ our mutual insurance rule is no more optimal.
} 
A pivotal agent, associated with coalition $S_{j}^{*}$, is by the consecutivity property, the most risky agent belonging to this club. He is the ultimate agent for which the net effect of his inclusion in the club is beneficial for all other (less risky) agents belonging to the club. Even though he increases the sum of risks in the club (i.e. the numerator of the risk premium), thus inflicting a loss to their welfare, his addition also increases its size (the denominator of the risk premium). Actually, his inclusion decreases the risk premium paid by each member of the coalition $S_{j}^{*}$. But if this coalition were to include the next agent, $p_{j}+1$, as he is more risky than $p_{j}$, the net effect of his inclusion would be negative for all other agents of $S_{j}^{*}$. Therefore they prefer not to let him in. In brief, adding the pivotal agent $p_{j}$ generates the lowest possible risk premium paid by each member of the coalition $S_{j}^{*}$.

Let us remark that the definition of a pivotal agent depends neither on the level of the variance nor on the degree of risk aversion. The conditions (7) and (8) can be rewritten as:

$$
1 \leq \frac{\left[2 n_{j}-1\right]}{\left(n_{j}-1\right)^{2}} \sum_{k \in S_{j} \backslash\left\{p_{j}\right\}} \prod_{z=k+1}^{p_{j}-1} \frac{1}{\lambda_{z}}
$$

and

$$
1>\frac{\left[2 n_{j}+1\right]}{n_{j}^{2}} \sum_{k \in S_{j}} \prod_{z=k+1}^{p_{j}} \frac{1}{\lambda_{z}} .
$$

What matters in the formation of a coalition, is the relative risk heterogeneity measured by risk ratios. Consider the less risky agent, characterized by $\sigma_{1}^{2}$. If he forms a coalition, it is necessarily with a more risky agent. The best choice for him is agent 2 who adds the lowest increase in the common risk premium:

$$
\begin{aligned}
\pi(\{1,2\}) & =\frac{\alpha}{8}\left(\sigma_{1}^{2}+\sigma_{2}^{2}\right)=\frac{\alpha \sigma_{1}^{2}}{8}\left(1+\lambda_{2}\right) \\
& <\pi(\{1, i\})=\frac{\alpha}{8}\left(\sigma_{1}^{2}+\sigma_{i}^{2}\right)=\frac{\alpha \sigma_{1}^{2}}{8}\left(1+\prod_{k=2}^{i} \lambda_{k}\right), \forall i>2 .
\end{aligned}
$$

This formula makes clear that agent 1 prefers to form a coalition with agent 2 than with any other agent in society, because he is relatively closer to him in terms of risk. Eventually, what matters for agent 1 , is the sequence of risk ratios, that is the individual variances relative to his own. This reasoning can be generalized to any $n$-agent coalition so as to obtain the core partition.

Given the consecutivity property of the core partition, the coalition $S_{j}^{*}$ is fully defined by the two agents whose indices are $p_{j-1}+1$ and $p_{j}$. In other words, the core partition is defined by the set of pivotal agents. Then we are able to offer the following:

Proposition 4 The core partition is characterized by a set of $J$ pivotal agents indexed by $p_{j}$ satisfying (7) - (8) for $j=1, \ldots, J-1$ and $\sigma_{p_{J}}^{2}=\sigma_{N}^{2}$. 
Remark that the last coalition is peculiar. Its pivotal agent is per force agent $N$ who satisfies condition (7) and not condition (8). We refer to this ultimate coalition as the "residual" risk-sharing coalition.

Finally, Proposition 4 highlights that, depending on the risk-ratio schedule, the mutual insurance rule may lead to various risk-sharing groups. The grand coalition belonging to the core obtains if the relative risk heterogeneity is sufficiently limited, corresponding to a ratio $\sigma_{N}^{2} / \sigma_{1}^{2}$ sufficiently small.

\subsection{Particular Risk-Ratio Schedules.}

We have just emphasized the importance of the risk-ratio schedule $\Lambda$ characterizing a society $\mathbf{I}$ in the endogenous determination of the core partition of this society. In this subsection, we explore the link between patterns of the risk-ratio schedule and the characteristics of the core partition. This allows us to better understand how heterogeneity affects the way individuals congregate so as to share risk. Formally, we want to assess the impact of $\Lambda$ on the series of pivotal agents, i.e. on the number and size of risk-sharing coalitions.

We restrict the analysis to risk-ratio schedules with simple monotonicity properties: either the sequence of $\lambda_{i}$ increases, decreases or remains constant. We then offer the following

Proposition 5 If the risk-ratio schedule $\Lambda=\left\{\lambda_{2}, \lambda_{3}, \ldots, \lambda_{N}\right\}$ is such that:

i/ $\lambda_{i}=\lambda, \forall i=2, . . N$ then $n_{j}^{*}=n, \forall j=1, \ldots, J-1$.

ii/ $\lambda_{i} \leq \lambda_{i+1}, \forall i=2, . . N$ then $n_{j}^{*} \geq n_{j+1}^{*}, \forall j=1, \ldots, J-1$.

iii/ $\lambda_{i} \geq \lambda_{i+1}, \forall i=2, . . N$ then $n_{j}^{*} \leq n_{j+1}^{*}, \forall j=1, \ldots, J-1$.

This proposition makes clear that risk heterogeneity affects the core partition, that is the way agents collectively cope with risk. To understand this proposition, each individual makes his decision about membership with several principles in mind that we have previously uncovered. First, he prefers joining the least risky coalition; second, he prefers being joined by the less risky agents among those who are more risky than himself; third, when selecting (approving the admission of) any member in his coalition, he takes into consideration the ratio between his risk and the one of a potential entrant. Consecutivity, the ordering of coalition-risk premia, and the impact of risk ratios in determining the pivotal agent of any coalition are the key elements for understanding how a core partition relates to the risk-ratio schedule.

First, consider that the risk ratios are constant and equal to $\lambda$. From (9) and (10), we see that inequalities determining the pivotal agent are identical for any club $S_{j}$. It turns out that coalitions 
in the core partition have the same size. In fact, it amounts to say that with constant risk ratios individuals, while deciding to form a risk-sharing group, individuals face the same trade-off whatever the level of their exposure to risk.

Second, consider that risk ratio, $\lambda_{i}$, is increasing with the individual's index $i$. The condition determining $p_{2}$ implies higher values of the risk ratios than the one determining $p_{1}$. Hence, pondering the benefit of increasing size and the cost of bearing risk with agents farther in the distribution of risk, the size of $S_{2}^{*}$ turns out to be smaller than the size of $S_{1}^{*}$. Repeating the argument, we find that the succeeding club sizes decrease.

Third, the case where risk ratio $\lambda_{i}$ is decreasing with the rank of individuals is easily understood by using a similar argument. Now the cost of forming the second risk-sharing group is lower yielding its size to be higher than for the first group.

\subsection{Risk-Sharing Partitions and Aggregate Risk Premium.}

We aim to study the impact of an increase in risk, on the pattern of risk-sharing coalitions and on the resource cost of dealing with risk. We first define the aggregate risk premium:

Definition 5 The aggregate risk premium associated with the core partition $\mathcal{P}$ is defined as:

$$
\begin{aligned}
\bar{\pi}(\mathcal{P}) & =\frac{1}{N} \sum_{i=1}^{N} \pi_{i}=\frac{1}{N}\left(\sum_{j=1}^{J} n_{j} \pi\left(S_{j}\right)\right) \\
& =\frac{1}{N} \frac{\alpha}{2}\left(\sum_{j=1}^{J} \frac{1}{n_{j}} \sum_{k \in S_{j}} \sigma_{k}^{2}\right)+\frac{\alpha}{2} \sigma_{\nu}^{2}
\end{aligned}
$$

The aggregate risk premium is an indicator of the willingness to pay for risk coping, at the society level. From equation (11), it clearly depends on the core partition.

We should expect that an increase in individual risk should lead to a higher aggregate risk premium. This is obviously true if the coalition structure is taken as given. However this is not necessarily true when agents form their risk-sharing coalitions. It may happen that the change in the whole core partition leads to different risk-sharing arrangements, the outcome of which is to decrease the average risk premium.

This counter-intuitive result is proven in the following

Proposition 6 Consider two societies $\mathbf{I}$ and $\mathbf{I}^{\prime}$ with $\varepsilon_{i}$, respectively $\varepsilon_{i}^{\prime}$, the idiosyncratic risk of any individual $i$ in $\mathbf{I}$, respectively $\mathbf{I}^{\prime}$. Assuming that $\varepsilon_{i} S S$-Dominates $\varepsilon_{i}^{\prime}$ for every $i=1, \ldots, N$, then society $\mathbf{I}$ may be characterized by a higher aggregate risk premium than $\mathbf{I}^{\prime}$ :

$$
\bar{\pi}\left(\mathcal{P}^{\prime}\right)<\bar{\pi}(\mathcal{P})
$$


where $\mathcal{P}$ (resp. $\left.\mathcal{P}^{\prime}\right)$ is the core partition associated with $\mathbf{I}\left(\mathbf{I}^{\prime}\right)$.

Proposition 6 highlights the fact that if endogenous formation of risk-sharing groups is taken into consideration, we cannot claim that all individuals pay a higher risk premium in a more risky society. We consider the case where society $\mathbf{I}^{\prime}$ is associated with a lower number of risk-sharing coalitions than $\mathbf{I}$, even though agents face more risk (higher idiosyncratic variances) in $\mathbf{I}^{\prime}$ than in $\mathbf{I}$, because as stressed in Proposition 5, this society is characterized by less risk heterogeneity. Hence, in society $\mathbf{I}^{\prime}$, risk may be allocated in larger coalitions. In other words, in society $\mathbf{I}^{\prime}$, individuals have the possibility to mutualize risk on a larger scale. This leads that the sum of these risk premia may be lower in the more risky society and some individuals will pay lower risk premium and consume more in this society.

\subsection{Varying the size of society.}

Our framework allows us to address the impact of a variation of population, that is an increase of $N$ through the addition of new agents. We distinguish two ways of varying the size of society: replicating society or introducing individuals characterized by new risks (new values of $\sigma_{i}^{2}$ ). We shall prove that they lead to contrasting results.

We index the type of risk aversion by $z$, and denote by $\sigma^{2}(z)$ the value of the risk aversion $z$ type. If there are $Z$ types of risk aversions, we order them as follows $\sigma^{2}(z)<\sigma^{2}\left(z^{\prime}\right)$ for $z<z^{\prime}$. The set of risk aversion types writes

$$
\vec{\sigma}(\mathbf{I})=\left\{\sigma^{2}(1), \sigma^{2}(2), \ldots \sigma^{2}(Z)\right\}
$$

We denote by $f\left(\sigma^{2}(z)\right)$ the fraction of the $N$ individuals with the risk $\sigma^{2}(z)$. This allows us to distinguish between $\sigma_{i}^{2}$, the risk borne by agent $i$, and the $z$-th type of risk $\sigma^{2}(z)$. Here we consider consecutive coalitions as we know that they belong to the core. We are thus able to show that

Lemma 1 When an individual $i$ with risk $\sigma^{2}(z)$ is in $S$ all individuals with the same $\sigma^{2}(z)$ belong to $S$.

Therefore, the core partition is characterized by a set of pivotal types of agents, that is a set of pivotal risks $\sigma^{2}\left(\widetilde{p}_{j}\right)$.

For a given coalition $S$, we denote by $\vec{\sigma}(S)=\left\{\sigma^{2}(z)\right.$, for any $\left.z=1, \ldots, k \mid \forall \sigma_{i}^{2}=\sigma^{2}(z), i \in S\right\}$. We define the certainty-equivalent income for individual $i$ in group $S$, denoted by $\omega_{i}(S)$, as:

$$
\omega_{i}(S)=w_{i}-\frac{\alpha}{2} \frac{\sum_{\sigma^{2}(z) \in \vec{\sigma}(S)} f\left(\sigma^{2}(z)\right) \sigma^{2}(z)}{N\left(\sum_{\sigma^{2}(z) \in \vec{\sigma}(S)} f\left(\sigma^{2}(z)\right)\right)^{2}}-\frac{\alpha}{2} \sigma_{\nu}^{2} .
$$


Hence, individual $i$ while choosing her welfare-maximizing coalition compares for any $\omega_{i}(S)$ and $\omega_{i}\left(S^{\prime}\right)$. As, from (13), this comparison does not depend on $N$, we immediately obtain the following

Proposition 7 The core partition is defined by a set of pivotal risks which depends on the density function of the risk distribution but is invariant to $N$.

However this proposition states that the relative sizes of types as captured by the density function matter for the characterization of the core partition. This result is a direct consequence of the mutual insurance rule which implies that the risk premium is a weighted average of all risks in this coalition with weights capturing the relative size of a type within the coalition. It turns out that the trade-off between size and heterogeneity driving the formation of coalitions does not depend on $N$.

This proposition allows us to address the case of replication of society $\mathbf{I}$. Let us consider the integer $a$ and $a \mathbf{I}$ is the society derived from $\mathbf{I}$ in which there are $a N$ agents, i.e. $a$ copies of any individual $i$.

It is immediate to state that

Corollary 1 The replication of society I does not modify the set of pivotal risks.

As replication does not modify the density function, $f\left(\sigma^{2}(z)\right)$, the set of pivotal risks characterizing the core partition is unaffected. Of course, the size of any coalition $S_{j}^{*}$ increases proportionally with $a$. Replication reduces the risk premium in each coalition as any individual shares risk with more individuals belonging to his coalition. In the limit, replication allows to diversify completely risk leading individuals to bear only the cost of the uninsurable shock, as $\lim _{a \longrightarrow \infty} \pi\left(S_{j}^{*}\right)=\frac{\alpha}{2} \sigma_{\nu}^{2}$ for any $S_{j}^{*}$ in the core partition.

Let us now turn to the case where society is enlarged by the introduction of agents characterized by new exposures to risk, that is the introduction of new types of agents. This amounts to change both the size of the population $N$ and the distribution of risks. We start with the set of individual risk aversions $\vec{\sigma}_{I}=\left\{\sigma_{1}^{2}, \sigma_{2}^{2}, \ldots \sigma_{N}^{2}\right\}$ which generates the core partition $\mathcal{P}^{*}=\left\{S_{1}^{*}, \ldots, S_{j}^{*}, \ldots, S_{J}^{*}\right\}$. For simplicity, let us add an individual $\widehat{i}$ characterized by a new risk $\widehat{\sigma}^{2} \notin \vec{\sigma}_{I}$ and such that $\sigma_{p_{j}-1}^{2}<\widehat{\sigma}^{2}<\sigma_{p_{j}}^{2}$. We have the following

Proposition 8 Consider the addition to society of one individual $\widehat{i}$ characterized by a risk $\widehat{\sigma}^{2} \notin \vec{\sigma}_{I}$ and such that $\sigma_{p_{j}-1}^{2}<\widehat{\sigma}^{2}<\sigma_{p_{j}}^{2}$. Then, the individual with $\sigma_{p_{j}}^{2}$ may not belong to the coalition to which $\widehat{i}$ belongs.

The introduction of a new risk modifies inequalities (7) and (8) characterizing pivotal agents and thus may modify the set of pivotal agents, in contrast with what was obtained when agents were 
added to society without changing the set of types. Hence, individual $\widehat{i}$ belongs to the $j$ coalition which new pivotal agent denoted by $p_{j}^{\prime}$ can differ from the previous one, i.e. $p_{j}^{\prime} \leq p_{j}$. Further, this change in the boundary of the $j$ coalition can potentially lead to a change of the whole pattern of risk coalitions comprising individuals with risks such that $\sigma^{2}>\sigma_{p_{j}^{\prime}}^{2}$. Take for instance the risksharing coalition $S_{j+1}$. If the less risky individual of $S_{j+1}$ has a lower risk than individual $p_{j}+1$ then the trade-off between heterogeneity and size determining the membership in $S_{j+1}$ is modified. Potentially, this can lead to a pivotal agent $p_{j+1}^{\prime} \leq p_{j+1}$.

An intriguing consequence of both propositions is that an increase of population (either an increase of $N$ without changing the risk distribution or an increase of $N$ with an increase in the risk type) does not necessarily reduce the number of risk-sharing coalitions nor modify the set of pivotal risks. ${ }^{13}$ Therefore none of these ways of increasing the size of society which could be viewed as a way to diversify risk actually lead to the grand coalition.

\section{Empirical Implications of Risk-Sharing Group Formation.}

Let us comment on the empirical consequences of the segmentation of society into multiple risksharing groups on the extent of risk sharing.

\subsection{Partial Risk-Sharing.}

Partial risk sharing within society corresponds to the simultaneous rejection of perfect risk sharing and autarky (cf. Dercon and Krishnan, 2003).

Most empirical studies test for efficient risk-sharing by considering that the conditional expectation of individual consumption equals:

$$
\mathbb{E}\left(c_{i t} \mid \frac{Y_{t}^{\mathbf{I}}}{N}, y_{i t}\right)=\kappa_{i}+\beta_{i} \frac{Y_{t}^{\mathbf{I}}}{N}+\zeta_{i} y_{i t}
$$

with $Y_{t}^{\mathbf{I}} \equiv \sum_{i=1}^{N} y_{i t}$, and where $\beta_{i}$ and $\zeta_{i}$ obtain using properties of conditional expectations of multivariate normal distributions (Ramanathan, 1993):

$$
\begin{aligned}
& \beta_{i}= \frac{\operatorname{cov}\left(\frac{Y_{t}^{\mathbf{I}}}{N}, c_{i t}\right) \operatorname{var}\left(y_{i t}\right)-\operatorname{cov}\left(y_{i t}, c_{i t}\right) \operatorname{cov}\left(\frac{Y_{t}^{\mathbf{I}}}{N}, y_{i t}\right)}{\operatorname{var}\left(\frac{Y_{t}^{\mathbf{I}}}{N}\right) \operatorname{var}\left(y_{i t}\right)-\left[\operatorname{cov}\left(\frac{Y_{t}^{\mathbf{I}}}{N}, y_{i t}\right)\right]^{2}} \\
& \zeta_{i}=\frac{\operatorname{cov}\left(y_{i t}, c_{i t}\right) \operatorname{var}\left(\frac{Y_{t}^{\mathbf{I}}}{N}\right)-\operatorname{cov}\left(\frac{Y_{t}^{\mathbf{I}}}{N}, c_{i t}\right) \operatorname{cov}\left(\frac{Y_{t}^{\mathbf{I}}}{N}, y_{i t}\right)}{\operatorname{var}\left(y_{i t}\right) \operatorname{var}\left(\frac{Y_{t}^{\mathbf{I}}}{N}\right)-\left[\operatorname{cov}\left(\frac{Y_{t}^{\mathbf{I}}}{N}, y_{i t}\right)\right]^{2}} .
\end{aligned}
$$

\footnotetext{
${ }^{13}$ Excluding the case of $N$ tending to infinity.
} 
Equation (14) builds on the well known result that with CARA utility function, individual consumption at the optimum is a linear function of both global resources and individual income (see for instance Townsend, 1994).

Denoting by $\bar{\beta}_{\mathbf{I}} \equiv \frac{\sum_{i \in \mathbf{I}} \beta_{i}}{N}$ and $\bar{\zeta}_{\mathbf{I}} \equiv \frac{\sum_{i \in \mathbf{I}} \zeta_{i}}{N}$, we then offer the following

Lemma 2 Given a partition of the society, if we assume that individuals share risk optimally within coalitions, whatever the Pareto-optimal risk-sharing rule, we get

$$
\begin{gathered}
\lim _{N \longrightarrow+\infty} \bar{\beta}_{\mathbf{I}}=1-\frac{1}{\bar{n}_{J}} \\
\lim _{N \longrightarrow+\infty} \bar{\zeta}_{\mathbf{I}}=\frac{1}{\bar{n}_{J}}
\end{gathered}
$$

with $\bar{n}_{J}$ the average size of risk-sharing groups.

It turns out that risk-sharing group membership is a crucial determinant of the value of $\beta_{i}$ and $\zeta_{i}$. If the grand coalition is formed, $\bar{n}_{J}=N$ implying that $\lim _{N \longrightarrow+\infty} \bar{\beta}_{\mathbf{I}}=1$ and $\lim _{N \longrightarrow+\infty} \bar{\zeta}_{\mathbf{I}}=0$. On the opposite, if individuals decide to pool risk in smaller groups than the whole society $\mathbf{I}, \bar{n}_{J}<N$ implying that $\lim _{N \longrightarrow+\infty} \bar{\beta}_{\mathbf{I}} \neq 1$ and $\lim _{N \longrightarrow+\infty} \bar{\zeta}_{\mathbf{I}} \neq 0$. Most empirical studies assume that the relevant unit to test for efficient risk sharing is the grand coalition. This assumption may be inaccurate and may explain why the null hypothesis $\bar{\zeta}_{\mathbf{I}}=0$ is rejected.

We will thus consider that $\bar{\zeta}_{\mathbf{I}}$ measures the extent of risk sharing in this society. A higher $\bar{\zeta}_{\mathbf{I}}$ means that an individual on average benefits from lower risk sharing. This is congruent with the interpretation of the estimated value of $\zeta$ as a measure of the extent of risk sharing (see for instance Jalan and Ravallion, 1999, and Suri, 2009).

Characterizing the relationship between heterogeneity in risk exposure and the size of risksharing coalitions, our setup helps to understand the impact of heterogeneity on the extent of risk sharing. Lemma 1 allows us to prove the following

Proposition 9 For two societies $\mathbf{I}$ and $\mathbf{I}^{\prime}$, I being characterized by $\Lambda=\left\{\lambda_{2}, \lambda_{3}, \ldots, \lambda_{N}\right\}$ and $\mathbf{I}^{\prime}$ being characterized by $\Lambda^{\prime}=\left\{\lambda_{2}^{\prime}, \lambda_{3}^{\prime}, \ldots, \lambda_{N}^{\prime}\right\}$, if $\lambda_{i}<\lambda_{i}^{\prime}, \forall i=2, \ldots, N$, then the extent of risk sharing is higher in society $\mathbf{I}$ than in society $\mathbf{I}^{\prime}$.

This proposition highlights the crucial impact of risk heterogeneity on the allocation of risk in any society. In the more heterogenous society $\mathbf{I}^{\prime}$, individuals share risk in smaller coalitions, thus diminishing the extent of risk sharing.

Let us provide an intuition for the proof of Proposition 9 by taking the special case where $\lambda_{i}=\lambda_{i+1}=\lambda, \lambda_{i}^{\prime}=\lambda_{i+1}^{\prime}=\lambda^{\prime}$ and $\lambda<\lambda^{\prime}$ whatever $i=2, \ldots, N$. Consider agent 1 in society $\mathbf{I}$. 
Taking into account that membership only depends on the risk-ratio schedule, and pondering the trade-off between the benefit of size and the cost of higher marginal relative risk, agent 1 prefers being included in a (weakly) larger risk-sharing coalition in society $\mathbf{I}$ than in society $\mathbf{I}^{\prime}$. From Proposition 5, the agent following the first pivotal agent faces the same trade-off as agent 1. Hence the second club is of the same size than the first club, and consequently is of a larger size in society $\mathbf{I}$ than in society $\mathbf{I}^{\prime}$. Repeating the argument, we find that the number of non-residual clubs is (weakly) reduced in the core partition of society $\mathbf{I}$ compared to the core partition of society $\mathbf{I}^{\prime}$. The case of decreasing and increasing $\lambda_{i}$ s can similarly be dealt with.

Propositions 6 and 9 highlight that the aggregate risk premium index and the extent of risk sharing must be considered for the assessment of risk sharing when risk-sharing coalitions are endogenous. Take two societies $\mathbf{I}$ and $\mathbf{I}^{\prime}$ with any $\varepsilon_{i}$ SS-Dominating $\varepsilon_{i}^{\prime}$ and the risk-ratio schedule in $\mathbf{I}$ being characterized by higher risk ratios than the one in $\mathbf{I}^{\prime}$ such that the aggregate risk premium and the extent of risk sharing may both be higher in society $\mathbf{I}^{\prime}$. A larger extent of risk sharing is commonly considered as desirable when it is assumed that agents are risk averse. However, a higher aggregate risk premium is viewed as worse insurance outcome as it would lead to a lower certainty-equivalent income. Hence, reasoning on the variation of the aggregate risk premium or the extent of risk sharing separately would draw contradictory conclusions about the impact of an increase in risk on risk sharing performances, at the society level.

\subsection{Discussion.}

Our results suggest that risk heterogeneity rather than the level of risk is the crucial dimension to understand the allocation of risk in a society. A possible empirical investigation of our model would be to examine the impact of risk heterogeneity on the extent of risk sharing. In particular, our model predicts that an increase in $\lambda_{i}$ for some $i$ may lead to smaller risk-sharing coalitions diminishing the extent of risk sharing.

As in Dubois (2006), a first potential test of this result would be to see whether the null hypothesis $\zeta_{i}=0$ or $\bar{\zeta}_{\mathbf{I}}=0$ is more likely rejected for societies characterized by larger ratios between two individuals' attributes. The evidence obtained by Dubois (2006, p.25), studying the impact on risk sharing of heterogeneity as measured by the variance of risk aversion, gives some support to our analysis as he writes"Also the higher is average risk aversion, and the more heterogenous it is, the more likely full risk sharing is rejected".

Recent works use disaggregated data and apply dyadic regression analysis to estimate the probability of a link between a pair of individuals in a network (see Fafchamps and Lund, 2003, Fafchamps and Gubert, 2007, Arcand and Fafchamps, 2012). Typically, dyadic regressions estimate the impact 
of a difference in attributes as well as of the sum of attributes on the likelihood of a link. The difference in attributes allows to identify the type of matching (positive or negative) while the sum of attributes captures the propensity of an individual to join a group conditional on characteristics. These works find evidence of assortative mating.

Building on their approach, one can envision testing the formation of risk-sharing coalitions. Our theory of the endogenous formation of coalitions when the mutual insurance rule is applied, suggests that consecutivity property be exploited. Namely, on the basis of equations (9) and (10) defining a pivotal agent, the probability of a link between two individuals $i$ and $i^{\prime}, i<i^{\prime}$, depends not only on the risk-ratio between individuals $i$ and $i^{\prime}$ but on the whole series of risk-ratios $\lambda_{z}$, for $z=i+1, \ldots, i^{\prime}$. For a given risk ratio $\sigma_{i^{\prime}}^{2} / \sigma_{i}^{2}$, the higher is the risk-ratio between any individual $z=i, i+1, \ldots, i^{\prime}-1$ and $i^{\prime}$ the lower the probability to create a link between $i$ and $i^{\prime}$. Introducing the whole series of risk-ratios in between two individuals as explanatory variables of the probability to create a link between them would be a way to test for endogenous formation of coalitions.

A further test of the consecutivity property would be to check whether, between three individuals such that $\sigma_{i}^{2}<\sigma_{i^{\prime}}^{2}<\sigma_{i^{\prime \prime}}^{2}$, the probability of a link between $i$ and $i^{\prime}$ is significantly higher than the probability of a link between $i$ and $i^{\prime \prime}$.

As a whole, our theoretical approach suggests that an empirical investigation of risk-sharing coalitions formation should take into account the whole risk-ratio schedule in society.

\section{Heterogenous Risk Aversion.}

Recent advances in the empirical literature support the evidence of heterogenous risk preferences (see for instance Ogaki and Zhang, 2001, Mazzocco, 2004, Dubois, 2006, Mazzocco and Saini, 2010, Chiappori et alii, 2011). In this section, we study the impact of heterogenous risk aversion on the formation of risk-sharing groups and show how the properties of the partition previously found remain valid.

The model we study is similar to the above setting with two modifications: First, individuals face the same exposure to risk $\sigma_{\varepsilon}^{2}$; second, individuals differ with respect to risk aversion. Without loss of generality, we index individuals as follows: for $i$ and $i^{\prime}=1, \ldots, N$ with $i>i^{\prime}$ then $\frac{1}{\alpha_{i}}>\frac{1}{\alpha_{i^{\prime}}}$. The inverse of risk aversion $\frac{1}{\alpha}$ being defined as risk tolerance, a lower indexed individual is characterized by a lower risk tolerance.

Considering the consumption function given by equation (2), under these assumptions, the 
indirect utility function for individual $i$ in $S_{j}$ obtains:

$$
V_{i}\left(S_{j}\right)=-\frac{1}{\alpha_{i}} e^{-\alpha_{i}\left[w_{i}-\frac{1}{2} \frac{1}{\alpha_{i}} \frac{n_{j}^{2}}{\left(\sum_{k \in S} \frac{1}{\alpha_{k}}\right)^{2}}\left(\sigma_{\nu}^{2}+\frac{\sigma_{\varepsilon}^{2}}{n_{j}}\right)\right]} .
$$

We denote by $\pi_{i}\left(S_{j}\right)$ the risk premium evaluated by individual $i$ when belonging to $S_{j}$ :

$$
\pi_{i}\left(S_{j}\right)=\frac{\alpha_{i}}{2}\left(\frac{\frac{1}{\alpha_{i}}}{\frac{1}{n_{j}} \sum_{z \in S_{j}} \frac{1}{\alpha_{z}}}\right)^{2}\left(\sigma_{\nu}^{2}+\frac{\sigma_{\varepsilon}^{2}}{n_{j}}\right) .
$$

The risk premium paid by agent $i$ in her risk-sharing group expresses the trade-off on which the partition of society is based. Accepting a new member in a group pools risk among more individuals and therefore reduces the risk associated with the group (which is equal to $\sigma_{\nu}^{2}+\frac{\sigma_{\varepsilon}^{2}}{n_{j}}$ in equation 16). However accepting a new member affects the coalition average risk tolerance: if this new member is less tolerant to risk than the average agent in the group, it will decrease the average risk tolerance and thus increase the transfer in order to be insured against risk. This is captured by the ratio $\frac{1}{\frac{1}{n_{j}} \sum_{z \in S_{j}}^{1} \frac{1}{\alpha_{z}}}$ in equation (16).

Defining the risk tolerance ratio between agent $i$ and agent $i-1$ as $\chi_{i}=\frac{1 / \alpha_{i}}{1 / \alpha_{i-1}}$ for any $i=2, \ldots, N$, the following proposition characterizes the core partition:

Proposition 10 A core partition $\mathcal{P}^{*}=\left\{S_{1}^{*}, \ldots, S_{j}^{*}, \ldots, S_{J}^{*}\right\}$ exists and is characterized as follows:

i/ It is unique if

$$
\forall z=2, \ldots, N-1, \quad \frac{\chi_{z+1}-\chi_{z}}{\chi_{z+1}-1} \geq-\frac{1}{(z+1)}
$$

ii/ It is consecutive, that is, if $i$ and $\widetilde{i}$ both belong to $S_{j}^{*}$ then $\forall i^{\prime}, i>i^{\prime}>\widetilde{i}, i^{\prime} \in S_{j}^{*}$.

iii/ For any two individuals $i \in S_{j}^{*}$ and $i^{\prime} \in S_{j^{\prime}}^{*}$ such that $\frac{1}{\alpha_{i}}>\frac{1}{\alpha_{i^{\prime}}}$, then $\pi_{i}\left(S_{j}^{*}\right) \leqq \pi_{i^{\prime}}\left(S_{j^{\prime}}^{*}\right)$.

This proposition is quite similar to Proposition 3. The sufficient condition for uniqueness parallels equation (6). Consecutivity is a characteristic of the core partition: two individuals are more likely to congregate the closer they are in terms of risk tolerance. Finally, the less risk tolerant an individual, the higher the risk premium that this individual is ready to pay. In other words, we can index coalitions according to the ordering of risk premia.

Notice however that the rationale behind the formation of coalitions and therefore of the core partition is different than in the case of heterogeneity with respect to risk. In the case of risk aversion heterogeneity, each agent wants to join a coalition formed by the most risk tolerant agents: This decreases her risk premium. But the most risk tolerant agents deny membership to agents 
with sufficiently high risk aversion who would demand a too high transfer. Hence the formation of the core partition is obtained by clustering the most risk tolerant agents into the first coalition, and proceeding sequentially for the other coalitions.

The core partition can be characterized by a set of pivotal agents defined as follows: ${ }^{14}$

$$
\sum_{k \in S_{j} \backslash\left\{p_{j}\right\}} \frac{1}{\alpha_{k}}\left(-1+\frac{1}{\left(n_{j}-1\right)} \sqrt{n_{j}^{2} \frac{\left(\sigma_{\nu}^{2}+\frac{\sigma_{\varepsilon}^{2}}{n_{j}}\right)}{\left(\sigma_{\nu}^{2}+\frac{\sigma_{\varepsilon}^{2}}{n_{j}-1}\right)}}\right) \leq \frac{1}{\alpha_{p_{j}}}
$$

and

$$
\sum_{k \in S_{j}} \frac{1}{\alpha_{k}}\left(-1+\frac{1}{n_{j}} \sqrt{\left(n_{j}+1\right)^{2} \frac{\left(\sigma_{\nu}^{2}+\frac{\sigma_{\varepsilon}^{2}}{n_{j}+1}\right)}{\left(\sigma_{\nu}^{2}+\frac{\sigma_{\varepsilon}^{2}}{n_{j}}\right)}}\right)>\frac{1}{\alpha_{p_{j}+1}} .
$$

As inequalities defining pivotal agents are homogenous of degree 0 with respect to $\frac{1}{\alpha_{i}}$, we also derive a relationship between the heterogeneity of risk tolerance and coalition's size:

Proposition 11 If the risk-tolerance ratio $\chi=\left\{\chi_{2}, \chi_{3}, \ldots, \chi_{N}\right\}$ is such that:

i) $\chi_{i}=\chi, \forall i=2, \ldots, N$, then $n_{j}^{*}=n, \forall j=1, \ldots, J-1$.

ii) $\chi_{i} \leq \chi_{i+1}, \forall i=2, \ldots, N$, then $n_{j}^{*} \leq n_{j+1}^{*}, \forall j=1, \ldots, J-1$.

iii) $\chi_{i} \geq \chi_{i+1}, \forall i=2, \ldots, N$, then $n_{j}^{*} \geq n_{j+1}^{*}, \forall j=1, \ldots, J-1$.

This proposition is similar to Proposition 5 and can be explained along the same line. What matters for the shaping of the partition is the heterogeneity with respect to risk aversion, captured by the risk tolerance ratios, which plays the same role as the risk ratios. ${ }^{15}$

In brief, our various propositions are robust to the nature of heterogeneity. This heterogeneity may apply to the utility functions of agents or to the stochastic environment they face, yet it will trigger identical behaviors which will lead agents to sort themselves into distinct risk-sharing groups (even though it may happen that the grand coalition forms). Importantly, segmentation of society into different risk-sharing coalitions does not depend on the levels of idiosyncratic characteristics such as the exposures to risk or risk aversion coefficients, but on heterogeneity as expressed by the risk ratios or the risk-tolerance ratios.

${ }^{14}$ Let us consider $\Omega\left(\sigma_{\nu}^{2}\right)=\left(-1+\frac{1}{n_{j}} \sqrt{\left(n_{j}+1\right)^{2} \frac{\left(\sigma_{\nu}^{2}+\frac{\sigma_{\varepsilon}^{2}}{n_{j}+1}\right)}{\left(\sigma_{\nu}^{2}+\frac{\sigma_{\varepsilon}^{2}}{n_{j}}\right)}}\right)$. It is easy to check that it monotonously increases with $\sigma_{\nu}^{2}, \forall n_{j} \geq 1$ and $0<\Omega(0)=\left(-1+\frac{1}{n_{j}} \sqrt{\left(n_{j}+1\right) n_{j}}\right)<1$ and $\lim _{\sigma_{\nu}^{2} \rightarrow+\infty} \Omega\left(\sigma_{\nu}^{2}\right)=\frac{1}{n_{j}}$.

${ }^{15} \mathrm{It}$ is irrelevant to search for a proposition equivalent to Proposition 4. Comparing aggregate risk premia obtained in societies which are not similar in risk aversion and thus value differently the protection with respect to risk has no economic meaning. 


\section{Conclusion.}

Non-financial risk-sharing arrangements are widely used in developing economies. In the absence of proper and well-functioning financial markets, agents rely on informal insurance schemes, often based on social or geographical (a "village") proximity. Hence it is legitimate to ask how risk-sharing mechanisms are designed in a society and what are their properties and consequences.

Considering a society without financial markets and relying on a particular insurance rule, we study the endogenous formation of risk-sharing coalitions. Agents can form any possible group but commit to remaining in their chosen group whatever the realization of idiosyncratic shocks.

We show that when heterogenous individuals can freely choose their risk-sharing coalitions then commitment to a Pareto-optimal allocation of resources does not inevitably imply perfect risk sharing as the grand coalition does not always form.

Then considering the mutual insurance rule, we successively study the cases where individuals are heterogeneous with respect to the variances of the idiosyncratic shocks, and their risk aversion. First we obtain a characterization of the core partition of society with respect to risk, depending on the differentiated idiosyncratic risks born by individuals. It is unique (under some mild assumption), and consecutive: a coalition integrates agents of relatively similar risks. There is perfect risk-sharing within a coalition. However, there is no full insurance across society. In other words, the amplitude of risk sharing cannot be studied without precisely taking into account the memberships of risk-sharing groups and their differences.

Turning to a discussion of the role of risk heterogeneity on the segmentation of society and focusing on three special cases, we characterize the relationship between the characteristics (i.e. number, sizes and memberships) of risk-sharing coalitions and the distribution of risk across society.

When the partitions into risk-sharing coalitions of two societies differing in their risk heterogeneity are compared, we prove that the extent of risk sharing captured by the average size of coalitions decreases with this heterogeneity. The link between partial risk sharing and risk heterogeneity comes from the partition of society into different risk-sharing coalitions shaped by relative risk heterogeneity. A more risky society (in the sense of second-order stochastic dominance) may devote less resources to risk sharing than a less risky one as it may be less heterogenous, thus less segmented, and therefore better able to pool individual risks. This illustrates a tension between the levels of individual variances and their ratios (which express relative risk heterogeneity). Finally, we examine two ways of increasing the size of society and show that even though conducive to risk diversification they do not lead to perfect risk sharing. When heterogeneity with respect to risk aversion is considered, similar propositions obtain. 
The present research proves how coalition theory tools can be applied to study the functioning of an economy in the presence of uncertainty when agents are risk-averse. It can be extended along several lines, where these tools are also of potential interest.

First, the assumption of full commitment could be relaxed so as to assess the impact of defection on the number and size of risk-sharing coalitions forming the core partition.

Second, our results on segmentation and partial risk sharing rely on the assumption that individuals form groups expecting the the sharing rule to be applied, possibly through bargaining. It would be worthwhile to study the impact of bargaining simultanously on both coalitions and transfers on the core partition.

Third, our paper shows that sorting individuals into risk-sharing coalitions affects the extent of risk sharing over the whole society. This suggests to empirically search for boundaries of groups as an explanatory link between society's heterogeneity and the degree of partial risk sharing. 


\section{References}

[1] Arcand, J.-L., and M. Fafchamps, 2012, "Matching in Community-Based Organizations", Journal of Development Economics, 98, 203-221.

[2] Arrow, K., 1964, "The Role of Securities in the Optimal Allocation of Risk Bearing", Review of Economic Studies, 31, 91-96.

[3] Atkeson, A., and R.E. Lucas, 1992, "On Efficient Distribution with Private Information", Review of Economic Studies, 59, 427-453.

[4] Attanasio, O., and S. Davis, 1996, "Relative Wage Movements and the Distribution of Consumption", Journal of Political Economy, 104, 1227-1262.

[5] Banerjee, S., H. Konishi and T. Sönmez, 2001, "Core in a Simple Coalition Formation Game", Social Choice and Welfare, 18, 135-153.

[6] Barr, A., Attanasio, O., Cardenas, J.C., and C. Meghir, 2011, "Risk Pooling, Risk Preferences, and Social Networks", American Economic Journal: Applied Economics, forthcoming.

[7] Baton, B., and J. Lemaire, 1981, "The Core of a Reinsurance Market", Astin Bulletin, 12, $57-71$.

[8] Bold, T., 2009, "Implications of Endogenous Group Formation for Efficient Risk Sharing", The Economic Journal, 119, 562-591.

[9] Borch, R., 1962, "Equilibrium in a Reinsurance Market", Econometrica, 30, 424-444.

[10] Bramoullé, Y., and R. Kranton, 2007, "Risk-Sharing Networks", Journal of Economic Behavior and Organization, 64, 275-294.

[11] Chiappori, P.-A., and Ph. Reny, 2005, "Matching to Share Risk", mimeo.

[12] Chiappori, P.-A., Samphantharak, K., Schulhofer-Wohl, S., and R. Townsend, 2011, "Heterogeneity and Risk Sharing in Village Economies", Federal Reserve Bank of Minneapolis, Working Paper 683.

[13] Cochrane, J., 1991, "A Simple Test of Consumption Insurance", Journal of Political Economy, 99, 957-976.

[14] Conley, T., and Ch. Udry, 2010, "Learning About a New Technology: Pineapple in Ghana", American Economic Review, 100, 35-69. 
[15] Deb, R., and T. Suri, 2012, "Endogenous Emergence of Credit Markets: Contracting in Response to a New Technology in Ghana", Journal of Development Economics, forthcoming.

[16] Dercon, S., 2004, "Income Risk, Coping Strategies and Safety Nets", in S. Dercon (Ed.), Insurance against Poverty, Oxford University Press, Oxford: U.K..

[17] Dercon S., and P. Krishnan, 2003,"Risk Sharing and Public Transfers", The Economic Journal, 113, C86-C94.

[18] Dercon, S., J. De Weerdt, T. Bold, and A. Pankhurst, 2006, "Group-Based Funeral Insurance in Ethiopia and Tanzania", World Development, 34, 685-703.

[19] Dubois, P., 2002, "Consommation, partage de risque et assurance informelle : développements théoriques et tests empiriques récents", L'Actualité économique, 116-149.

[20] Dubois, P., 2006, "Heterogeneity of Preferences, Limited Commitment and Coalitions: Empirical Evidence on the Limits to Risk Sharing in Rural Pakistan", mimeo.

[21] Dubois, P., B. Jullien, and Th. Magnac, 2008, "Formal and Informal Risk Sharing in LDCs: Theory and Empirical Evidence", Econometrica, 76, 679-725.

[22] Fafchamps, M., 2008, "Risk Sharing Between Households", in J. Benhabib, A. Bisin and M. Jackson eds, Handbook of Social Economics, Elsevier.

[23] Fafchamps, M., and S. Lund, 2003, "Risk-Sharing Networks in Rural Philippines", Journal of Development Economics, 71, 261-287.

[24] Farrell, J., and S. Scotchmer, 1988,"Partnerships", Quarterly Journal of Economics, 103, 279297.

[25] Genicot, G., 2006, "Does Wealth Inequality Help Informal Insurance?", mimeo.

[26] Genicot, G., and D. Ray, 2003, "Group Formation in Risk-Sharing Arrangements", Review of Economic Studies, 40, 87-113.

[27] Ghatak, M., 1999, "Group Lending, Local Information and Peer Selection", Journal of Development Economics, 60, 27-53.

[28] Hayashi, F., J. Altonji and L. Kotlikoff, 1996, "Risk Sharing between and within Families", Econometrica, 64, 261-294. 
[29] Henriet, D., and J.-Ch. Rochet, 1987, "Some Reflections on Insurance Pricing", European Economic Review, 31, 863-885.

[30] Jalan, J., and M. Ravallion, 1999, "Are the Poor Less Well Insured? Evidence on Vulnerability to Income Risk in Rural China", Journal of Development Economics, 58, 61-81.

[31] Kazianga, H., and Ch. Udry, 2005, "Consumption Smoothing ? Livestock, Insurance and Drought in Rural Burkina Faso", mimeo.

[32] Kocherlakota, N., 1996, "Implications of Efficient Risk Sharing Without Commitment", Review of Economic Studies, 63, 595-609.

[33] Legros, P., and A. Newman, 2007, "Beauty is a Beast, Frog is a Prince: Assortative Matching with Nontransferabilities", Econometrica, 75, 1073-1102.

[34] Ligon, E., 1998, "Risk Sharing and Information in Village Economies", Review of Economic Studies, 65, 847-864.

[35] Ligon, E., Thomas, J., and T. Worrall, 2002, "Mutual Insurance and Limited Commitment: Theory and Evidence in Village Economies", Review of Economic Studies, 69, 115-139.

[36] Mace, B., 1991,"Full Insurance in the Presence of Aggregate Uncertainty", Journal of Political Economy, 99, 928-956.

[37] Mazzocco, M., 2004, "Savings, Risk Sharing and Preferences for Risk", American Economic Review, 94, 1169-1182.

[38] Mazzocco, M., and S. Saini, 2010, "Testing Efficient Risk Sharing with Heterogenous Risk Preferences", American Economic Review, forthcoming.

[39] Ogaki, M., and Q. Zhang, 2001, "Decreasing Relative Risk Aversion and Tests of Risk Sharing", Econometrica, 69, 515-526.

[40] Ramanathan, R., Statistical Methods in Econometrics, Academic Press, 1993.

[41] Ray, D., Development Economics, Princeton University Press, 1998.

[42] Suri, T., 2009, "Spillovers in Village Consumption: Testing the Extent of Partial Insurance", mimeo.

[43] Taub, B., and H. Chade, 2002, "Segmented Risk in a Continuous-Time Setting", Economic Theory, 20, 645-675. 
[44] Thomson, W., 1994, "Cooperative Models of Bargaining", in R.J. Aumann and S. Hart (Eds.), Handbook of Game Theory, vol. 2, 1237-1284, North Holland, Amsterdam.

[45] Townsend, R., 1994, "Risk and Insurance in Village India", Econometrica, 62, 539-591.

[46] Townsend, R., 1995, "Consumption Insurance: An Evaluation of Risk-Bearing Systems in LowIncome Economies", Journal of Economic Perspectives, 9, 83-102.

[47] Wilson, R., 1968, "The Theory of Syndicates", Econometrica, 36, 119-132. 


\section{Appendix.}

\subsection{Proof of Proposition 1.}

Let us consider, for any $S \subseteq \mathbf{I}$, the Pareto-optimal consumptions obtained in the optimal program:

$$
c_{i}(S)=\frac{1}{\alpha_{i}}\left[\ln \mu_{i}(S)-\frac{\sum_{k \in S} \frac{\ln \mu_{k}(S)}{\alpha_{k}}}{\sum_{k \in S} \frac{1}{\alpha_{k}}}\right]+\frac{\frac{1}{\alpha_{i}}}{\sum_{k \in S} \frac{1}{\alpha_{k}}} \sum_{k \in S}\left(w_{k}+\varepsilon_{k}+\nu\right), \forall i \in S
$$

Given that $\mathbb{E}\left[\varepsilon_{k}\right]=0$ and letting $n \equiv \operatorname{card}(S)$ we have

$$
\mathbb{E}\left[c_{i}(S)\right]=\frac{1}{\alpha_{i}}\left[\ln \mu_{i}(S)-\frac{\sum_{k \in S} \frac{\ln \mu_{k}(S)}{\alpha_{k}}}{\sum_{k \in S} \frac{1}{\alpha_{k}}}\right]+\frac{\frac{1}{\alpha_{i}}}{\sum_{k \in S} \frac{1}{\alpha_{k}}} \sum_{k \in S} w_{k}
$$

and

$$
\operatorname{Var}\left[c_{i}(S)\right]=\left(\frac{\frac{1}{\alpha_{i}}}{\sum_{k \in S} \frac{1}{\alpha_{k}}}\right)^{2}\left(\sum_{k \in S} \sigma_{k}^{2}+n \sigma_{\nu}^{2}\right)
$$

Hence, the certainty-equivalent income for any individual $i$ in $S$ writes as follows:

$$
\omega_{i}(S)=\frac{1}{\alpha_{i}}\left(\ln \mu_{i}(S)-\frac{\sum_{k \in S} \eta_{k}(S)}{\sum_{k \in S} \frac{1}{\alpha_{k}}}\right),
$$

where

$$
\eta_{k}(S) \equiv \frac{\ln \mu_{k}(S)}{\alpha_{k}}-\left(w_{k}-\frac{1}{2} \frac{\sigma_{k}^{2}+\sigma_{\nu}^{2}}{\sum_{m \in S} \frac{1}{\alpha_{m}}}\right) .
$$

We have for any $S$ and $S^{\prime} \subseteq \mathbf{I}, S \neq S^{\prime}$, any $i$ in $S$ and $S^{\prime}$,

$$
V_{i}(S) \gtreqless V_{i}\left(S^{\prime}\right)
$$

which is equivalent to

$$
\ln \mu_{i}(S)-\ln \mu_{i}\left(S^{\prime}\right) \gtreqless \frac{\sum_{k \in S} \eta_{k}(S)}{\sum_{k \in S} \frac{1}{\alpha_{k}}}-\frac{\sum_{k \in S^{\prime}} \eta_{k}\left(S^{\prime}\right)}{\sum_{k \in S^{\prime}} \frac{1}{\alpha_{k}}} .
$$

Following Banerjee, Konishi and Sönmez (2001), the common ranking property is defined as follows:

Definition 6 A coalition formation game $G$ satisfies the common ranking property if and only if there exists an ordering $\succeq$ over the set of all possible coalitions in $\mathbf{I}$ such that for any $i \in \mathbf{I}$ and any coalition $S, S^{\prime}$ in the set of all coalitions containing $i$ we have $S \succeq_{i} S^{\prime} \Leftrightarrow S \succeq S^{\prime}$, with $\succeq_{i}$ a preference relation of $i$ in the set of all coalitions containing $i$. 
We deduce that the common ranking condition is satisfied when

$$
\ln \mu_{i}(S)-\ln \mu_{i}\left(S^{\prime}\right)=\ln \mu_{j}(S)-\ln \mu_{j}\left(S^{\prime}\right), \forall i, j \text { in } S \text { and } S^{\prime} .
$$

It is immediate to see that this equation is satisfied under Assumption 1.

The Baton-Lemaire Rule. Under Assumption 2, we have

$$
\eta_{k}(S)=0, \forall k \in S
$$

which implies that (18) can be written as follows

$$
\frac{\alpha_{i}}{2}\left(-\frac{\sigma_{i}^{2}+\sigma_{\nu}^{2}}{\sum_{m \in S} \frac{1}{\alpha_{m}}}\right)+\frac{\alpha_{i}}{2}\left(\frac{\sigma_{i}^{2}+\sigma_{\nu}^{2}}{\sum_{m \in S^{\prime}} \frac{1}{\alpha_{m}}}\right) \gtreqless 0
$$

which is equivalent to

$$
\frac{1}{\sum_{m \in S^{\prime}} \frac{1}{\alpha_{m}}} \gtreqless \frac{1}{\sum_{m \in S} \frac{1}{\alpha_{m}}}
$$

which does not depend on any characteristics of individual $i$. Hence, the result. Remark that when $\alpha_{i}=\alpha$ for any $i \in \mathbf{I}(19)$ can be written as follows

$$
\frac{1}{n^{\prime}} \gtreqless \frac{1}{n}
$$

implying that $S$ is preferred to $S^{\prime}$ when it is larger than $S^{\prime}$. Hence, the grand coalition belongs to the core of the coalition formation game.

A risk-sharing rule obtained by Nash Bargaining. Assumption 1 allows us to consider the case where $\frac{\ln \mu_{i}(S)}{\alpha_{i}}=w_{i}-\frac{\alpha_{i}}{2} \sigma_{i}^{2}$ for any $i \in \mathbf{I}$ leading to the following

$$
c_{i}(S)=w_{i}-\frac{\alpha_{i}}{2} \sigma_{i}^{2}+\frac{\frac{1}{\alpha_{i}}}{2 \sum_{k \in S} \frac{1}{\alpha_{k}}} \sum_{k \in S}\left(\alpha_{k} \sigma_{k}^{2}\right)+\frac{\frac{1}{\alpha_{i}}}{\sum_{k \in S} \frac{1}{\alpha_{k}}} \sum_{k \in S}\left(\varepsilon_{k}+\nu\right), \forall i \in S .
$$

Let us consider that individuals in a given coalition $S$ bargain over the allocation of resources available in $S$. Denoting by $\epsilon$ the state of nature which belongs to a set $A, f($.$) the density function,$ $c_{i}(\epsilon)$ the level of consumption of individual $i$ under $\epsilon$ and $c_{i}(d)$ consumption under disagreement, the Nash bargaining problem writes (see Thomson, 1994)

$$
\max _{c_{i}(\epsilon)} \prod_{i \in S}\left(\int_{A} u\left(c_{i}(a)\right) f(\epsilon) d \epsilon-\int_{A} u\left(c_{i}(d)\right) f(\epsilon) d \epsilon\right)
$$

subject to the following resource constraint

$$
\sum_{i \in S} c_{i}(\epsilon)=\sum_{i \in S} y_{i}(\epsilon), \forall \epsilon \in A
$$


We know that the Nash bargaining solution is Pareto optimal, hence risk sharing is optimal between individuals belonging to coalition $S$.

Applying the log function and assuming that utility is CARA we have

$$
\max _{c_{i}(\epsilon)} \sum_{i \in S} \ln \left(\int_{A} \frac{1}{\alpha} e^{-\alpha c_{i}(d)} f(\epsilon) d \epsilon-\int_{A} \frac{1}{\alpha} e^{-\alpha c_{i}(\epsilon)} f(\epsilon) d \epsilon\right)
$$

subject to the following resource constraint

$$
\sum_{i \in S} c_{i}(\epsilon)=\sum_{i \in S} y_{i}(\epsilon), \forall \epsilon \in A
$$

Denoting by $\Psi(\epsilon)$ the Lagrange multiplier associated to the resource constraint, the first order condition is written as follows:

$$
\frac{e^{-\alpha_{i} c_{i}(\epsilon)}}{\left(\int_{A} \frac{1}{\alpha_{i}} e^{-\alpha_{i} c_{i}(d)} f(\epsilon) d \epsilon-\int_{A} \frac{1}{\alpha_{i}} e^{-\alpha_{i} c_{i}(\epsilon)} f(\epsilon) d \epsilon\right)}=\Psi(\epsilon), \forall \epsilon \in A, \forall i \in S
$$

Integrating both sides of (21) we find that

$$
\frac{\int_{A} e^{-\alpha_{i} c_{i}(\epsilon)} f(\epsilon) d \epsilon}{\left(\int_{A} e^{-\alpha c_{i}(d)} f(\epsilon) d \epsilon-\int_{A} e^{-\alpha c_{i}(\epsilon)} f(\epsilon) d \epsilon\right)}=\frac{1}{\alpha_{i}} \int_{A} f(\epsilon) \Psi(\epsilon) d \epsilon
$$

which is equivalent to

$$
\frac{e^{-\alpha_{i} \omega_{i}(S)}}{e^{-\alpha_{i} \omega_{i}(d)}-e^{-\alpha_{i} \omega_{i}(S)}}=\frac{1}{\alpha_{i}} \int_{A} f(\epsilon) \Psi(\epsilon) d \epsilon
$$

leading to

$$
\frac{\alpha_{i}}{e^{\alpha_{i}\left(\omega_{i}(S)-\omega_{i}(d)\right)}-1}=\int_{A} f(\epsilon) \Psi(\epsilon) d \epsilon .
$$

We thus deduce that for any $i, z$ in $S$

$$
\frac{\alpha_{i}}{e^{\alpha_{i}\left(\omega_{i}(S)-\omega_{i}(d)\right)}-1}=\frac{\alpha_{z}}{e^{\alpha_{z}\left(\omega_{z}(S)-\omega_{z}(d)\right)}-1}
$$

From (21) and (22) we have

$$
e^{-\left(\alpha_{i} c_{i}(\epsilon)-\alpha_{z} c_{z}(\epsilon)\right)}=\frac{e^{-\alpha_{i} \omega_{i}(S)}}{e^{-\alpha_{z} \omega_{z}(S)}}
$$

which yields

$$
c_{z}(\epsilon)=\frac{\alpha_{i}}{\alpha_{z}} c_{i}(\epsilon)+\omega_{z}(S)-\frac{\alpha_{i}}{\alpha_{z}} \omega_{i}(S) .
$$

Summing over all individuals $z$ in coalition $S$ and taking the feasibility constraint for coalition $S$ leads to

$$
c_{i}(S)=\omega_{i}(S)-\frac{\frac{1}{\alpha_{i}}}{\sum_{z \in S} \frac{1}{\alpha_{z}}} \sum_{z \in S} \omega_{z}(S)+\frac{\frac{1}{\alpha_{i}}}{\sum_{z \in S} \frac{1}{\alpha_{z}}} \sum_{z \in S}\left(w_{z}+\varepsilon_{z}+\nu\right)
$$


Assuming $\alpha_{i}=\alpha, \forall i \in \mathbf{I},(22)$ implies that

$$
\omega_{i}(S)-\omega_{i}(d)=\omega_{z}(S)-\omega_{z}(d)
$$

Assuming also that under disagreement the individual consumes is income $w_{i}+\varepsilon_{i}$ leading to the following certainty-equivalent income under disagreement

$$
\omega_{i}(d)=w_{i}-\frac{1}{2} \alpha\left(\sigma_{i}^{2}+\sigma_{\nu}^{2}\right)
$$

(24) becomes

$$
\omega_{z}(S)=\omega_{i}(S)-\left(w_{i}-\frac{1}{2} \alpha \sigma_{i}^{2}\right)+w_{z}-\frac{1}{2} \alpha \sigma_{z}^{2}
$$

Introducing this equation in (23) and letting $n \equiv \operatorname{card}(S)$ we have

$$
c_{i}(S)=\omega_{i}(S)-\frac{1}{n_{j}} \sum_{z \in S}\left(\omega_{i}(S)-\left(w_{i}-\frac{1}{2} \alpha \sigma_{i}^{2}\right)+w_{z}-\frac{1}{2} \alpha \sigma_{z}^{2}\right)+\frac{1}{n_{j}} \sum_{z \in S}\left(w_{z}+\varepsilon_{z}+\nu\right)
$$

which leads to

$$
c_{i}(S)=w_{i}-\frac{1}{2} \alpha \sigma_{i}^{2}+\frac{1}{2} \frac{\alpha \sum_{z \in S} \sigma_{z}^{2}}{n}+\frac{\sum_{z \in S}\left(\varepsilon_{z}+\nu\right)}{n} .
$$

It is immediate to check that the solution of the Nash Bargaining Problem equals optimal consumption under the Baton and Lemaire given in (20a) when $\alpha_{i}=\alpha, \forall i \in \mathbf{I}$.

\subsection{Proposition 2.}

It is always possible to find $\mu_{z}$ and $\Gamma_{i}=\left(\sigma_{i}^{2}, w_{i}, \alpha_{i}\right)$ for any $i$ such that we have

$$
\ln \mu_{i}(S)-\frac{\sum_{k \in S} \eta_{k}(S)}{\sum_{k \in S} \frac{1}{\alpha_{k}}}>\ln \mu_{i}(\mathbf{I})-\frac{\sum_{k \in \mathbf{I}} \eta_{k}(\mathbf{I})}{\sum_{k \in \mathbf{I}} \frac{1}{\alpha_{k}}} \text { for } S \subset \mathbf{I} .
$$

For instance let us consider that $\Gamma_{i}=\left(\sigma_{i}^{2}, w, \alpha\right)$ for any $i$ and $\ln \mu_{z}=\ln \mu$ for any $i$. Let us consider that $S=\mathbf{I} \backslash\{z\}$. It is easy to find $\sigma_{i}^{2}$ such that

$$
\sigma_{i}^{2}>\frac{(2 N-1)}{N(N-1)^{2}} \sum_{k \in \mathbf{I} \backslash\{z\}} \sigma_{k}^{2}
$$

\subsection{Proof of Proposition 3.}

\section{Proof of (ii): Consecutivity.}

By contradiction, let us consider a core-partition $\mathcal{P}^{*}$ characterized by some non consecutive groups, that is, there exist individuals $i, \widetilde{i} \in S_{j}^{*}$ and $i^{\prime} \in S_{j^{\prime}}^{*}$ with $i<i^{\prime}<\widetilde{i}$.

Suppose first that $\pi\left(S_{j}^{*}\right) \geq \pi\left(S_{j^{\prime}}^{*}\right)$. As $i<i^{\prime}<\widetilde{i} \Longleftrightarrow \sigma_{i}^{2}<\sigma_{i^{\prime}}^{2}<\sigma_{\tilde{i}}^{2}$, we have $\pi\left(S_{j^{\prime}}^{*}\right)>$ $\pi\left(\left(S_{j^{\prime}}^{*} \backslash\left\{i^{\prime}\right\}\right) \cup\{i\}\right)$, which leads to

$$
\forall z \in\left(S_{j^{\prime}}^{*} \backslash\left\{i^{\prime}\right\}\right) \cup\{i\}, V_{z}\left(\left(S_{j^{\prime}}^{*} \backslash\left\{i^{\prime}\right\}\right) \cup\{i\}\right)>V_{z}\left(\mathcal{P}^{*}\right)
$$


Second, assume that $\pi\left(S_{j^{\prime}}^{*}\right) \geq \pi\left(S_{j}^{*}\right)$. We have $\left.\pi\left(S_{j}^{*}\right)>\pi\left(\left(S_{j}^{*} \backslash \widetilde{i}\right\}\right) \cup\left\{i^{\prime}\right\}\right)$, which leads to

$$
\forall z \in\left(S_{j}^{*} \backslash\{\widetilde{i}\}\right) \cup\left\{i^{\prime}\right\}, V_{z}\left(\left(S_{j}^{*} \backslash\{\widetilde{i}\}\right) \cup\left\{i^{\prime}\right\}\right)>V_{z}\left(\mathcal{P}^{*}\right) .
$$

Hence a contradiction with the fact that $\mathcal{P}^{*}$ is assumed to be a core-partition.

\section{Proof of (i): Uniqueness.}

Let us define $p_{j}$ the most risky agent of the consecutive group $S_{j} \backslash\left\{p_{j}\right\}$ with size $\widetilde{n}_{j}=n_{j}-1$ satisfying the two following inequalities:

$$
\sigma_{p_{j}}^{2} \leq\left[2 \widetilde{n}_{j}+1\right] \sum_{k \in S_{j} \backslash\left\{p_{j}\right\}} \frac{\sigma_{k}^{2}}{\widetilde{n}_{j}^{2}}
$$

and

$$
\sigma_{p_{j}+1}^{2}>\left[2 \widetilde{n}_{j}+3\right] \sum_{k \in S_{j}} \frac{\sigma_{k}^{2}}{\left(\widetilde{n}_{j}+1\right)^{2}} .
$$

Let us consider the consecutive group $S_{j}$ whose lowest-individual-risk agent is $i$. Given the definition of the most risky agent, we can introduce the two following functions: $\Gamma(\widetilde{n})=\frac{\widetilde{n}}{2 \widetilde{n}+1}$ and $\Theta(i, \widetilde{n})=$ $\frac{1}{\widetilde{n}} \frac{\sum_{k=i}^{i+\widetilde{n}-1} \sigma_{k}^{2}}{\sigma_{i+n}^{2}}$ with $\widetilde{n}=1, \ldots, N-i+1$. Let us denote by $\widetilde{n}^{*}(i)+1$ the size of group $S_{j}$ such that:

$$
\Gamma\left(\widetilde{n}^{*}(i)\right) \leq \Theta\left(i, \widetilde{n}^{*}(i)\right)
$$

and

$$
\Gamma\left(\widetilde{n}^{*}(i)+1\right)>\Theta\left(i, \widetilde{n}^{*}(i)+1\right)
$$

It is easy to check that $\Gamma(\widetilde{n})$ is an increasing function of $\widetilde{n}$ and $\Gamma(1)=\frac{1}{3}$. Given $\Theta(i, 1)=1>\Gamma(1)$, if $\Theta(i, \widetilde{n})$ is decreasing with respect to $\widetilde{n}$ whatever $i \in \mathbf{I}$ and $\widetilde{n} \leq N-i$, then $\widetilde{n}^{*}(i)$ is unique as $\Gamma(\widetilde{n}) \leq \Theta(i, \widetilde{n})$ for $\widetilde{n} \leq \widetilde{n}^{*}(i)$ and $\Gamma(\widetilde{n})>\Theta(i, \widetilde{n})$ for $\widetilde{n}>\widetilde{n}^{*}(i)$.

The function $\Theta(i, \widetilde{n}(i))$ is decreasing if and only if:

$$
\begin{aligned}
\Delta \Theta(i, \widetilde{n}) & \equiv \Theta(i, \widetilde{n}(i)+1)-\Theta(i, \widetilde{n}(i))=\frac{1}{\widetilde{n}+1} \frac{\sigma_{i+\widetilde{n}}^{2}+\sum_{k=i}^{i+\widetilde{n}-1} \sigma_{k}^{2}}{\sigma_{i+\widetilde{n}+1}^{2}}-\frac{1}{\widetilde{n}} \frac{\sum_{k=i}^{i+\widetilde{n}-1} \sigma_{k}^{2}}{\sigma_{i+\widetilde{n}}^{2}}<0 \Longleftrightarrow \\
\psi(i, \widetilde{n}) & =\widetilde{n} \sigma_{i+\widetilde{n}}^{2}-\left((\widetilde{n}+1) \frac{\sigma_{i+\widetilde{n}+1}^{2}}{\sigma_{i+\widetilde{n}}^{2}}-\widetilde{n}\right)\left(\sum_{k=i}^{i+\widetilde{n}-1} \sigma_{k}^{2}\right)<0 .
\end{aligned}
$$

Let us consider the function $\psi(i, \widetilde{n})$. It is negative for all $i, \widetilde{n} \leq N-i$ if

$$
\psi(i, 1)=\sigma_{i+1}^{2}-\left(2 \frac{\sigma_{i+2}^{2}}{\sigma_{i+1}^{2}}-1\right)\left(\sigma_{i}^{2}\right) \leq 0 \text { and } \Delta \psi(i, \widetilde{n}) \equiv \psi(i, \widetilde{n}+1)-\psi(i, \widetilde{n}) \leq 0 .
$$

Defining $\lambda_{i+1}=\frac{\sigma_{i+1}^{2}}{\sigma_{i}^{2}}$, the inequality $\psi(i, 1) \leq 0$ is equivalent to

$$
\frac{\left(\frac{\sigma_{i+1}^{2}-\sigma_{i}^{2}}{\sigma_{i}^{2}}\right)-\left(\frac{\sigma_{i+2}^{2}-\sigma_{i+1}^{2}}{\sigma_{i+1}^{2}}\right)}{\left(\frac{\sigma_{i+2}^{2}-\sigma_{i+1}^{2}}{\sigma_{i+1}^{2}}\right)}=\frac{\lambda_{i+1}-\lambda_{i+2}}{\lambda_{i+2}-1} \leq 1 .
$$


Moreover, $\forall \widetilde{n} \geq 1, \Delta \psi(i, \widetilde{n}) \leq 0$ is equivalent to

$$
\begin{gathered}
\Delta \psi(i, \widetilde{n})=\left((\widetilde{n}+1) \lambda_{i+\widetilde{n}+1}-(\widetilde{n}+2) \lambda_{i+\widetilde{n}+2}+1\right)\left(\sigma_{i+\widetilde{n}}^{2}+\left(\sum_{k=i}^{i+\widetilde{n}-1} \sigma_{k}^{2}\right)\right) \leq 0 \Leftrightarrow \\
\frac{\lambda_{i+\widetilde{n}+1}-\lambda_{i+\widetilde{n}+2}}{\lambda_{i+\widetilde{n}+2}-1}(\widetilde{n}+1) \leq 1 .
\end{gathered}
$$

Defining $z \equiv i+\widetilde{n}+1$, we can rewrite this inequality as follows:

$$
\frac{\lambda_{z}-\lambda_{z+1}}{\lambda_{z+1}-1}(z+1)\left(\frac{z-i}{z+1}\right) \leq 1 .
$$

As $0 \leq \frac{(z-i)}{(z+1)} \leq 1$, we deduce that if for all $z=3, \ldots, N-1, \frac{\lambda_{z}-\lambda_{z+1}}{\lambda_{z+1}-1}(z+1) \leq 1$, then $\triangle \psi(i, \widetilde{n}) \leq 0$. Given equation (25), we deduce that if for all $z=2, \ldots, N-1, \frac{\lambda_{z}-\lambda_{z+1}}{\lambda_{z+1}-1}(z+1) \leq 1$ then $\triangle \psi(i, \widetilde{n}) \leq 0$ and $\psi(i, 1) \leq 0, \forall i=1, \ldots, N$.

Hence, when for all $z=2, \ldots, N-1, \frac{\lambda_{z}-\lambda_{z+1}}{\lambda_{z+1}-1}(z+1) \leq 1$, we deduce that there is a unique size $n_{j}$ for the club $S_{j}$.

Proof of (iii): Risk premium ordering.

Consider the first group $S_{1}^{*}$. Let us define the group $£_{j}=\left\{1, \ldots, n_{j}^{*}\right\}$ which is consecutive, comprised of the lowest-individual-risk agents and has the same size as group $S_{j}^{*}$. From the definition of the core-partition, we know that, $\forall £ \subset \mathbf{I}, \forall z \in S_{1}^{*}$ and $£, V_{z}\left(S_{1}^{*}\right) \geq V_{z}(£)$ and in particular $\forall z \in$ $S_{1}^{*}$ and $£_{j}, \forall j=2, \ldots, J, V_{z}\left(S_{1}^{*}\right)>V_{z}\left(£_{j}\right)$ which means that $\forall £_{j}, \pi\left(S_{1}^{*}\right)<\pi\left(£_{j}\right)$. Moreover, given the consecutivity property, it is easy to show that $\pi\left(£_{j}\right)<\pi\left(S_{j}^{*}\right), \forall j>1$. Hence, $\pi\left(S_{1}^{*}\right)<\pi\left(S_{j}^{*}\right)$. Considering the subset $\mathbf{I} \backslash\left(S_{1}^{*} \cup S_{2}^{*} \cup \ldots \cup S_{j}^{*}\right)$, the same argument can be applied for $S_{j+1}^{*}$ leading to the result $\pi\left(S_{1}^{*}\right)<\pi\left(S_{2}^{*}\right)<\pi\left(S_{3}^{*}\right)<\ldots<\pi\left(S_{j}^{*}\right)<\ldots<\pi\left(S_{J-1}^{*}\right)$.

\subsection{Proof of Proposition 5.}

Let us first denote by $S^{c}(i)$ any consecutive group whose less risky individual is $i$. We will denote by $\widehat{n}(i)$ the size of $S^{c}(i)$ such that $\widehat{n}(i)=\arg \max V_{i}\left(S^{c}(i)\right)$ in the subset $\mathbf{I} \backslash\{1,2, \ldots, i-1\}$, for a risk ratio schedule $\Lambda$. Hence, $\widehat{n}(i)$ satisfies inequalities characterizing a pivotal agent:

$$
\Gamma(\widehat{n}(i)-1) \leq \Theta(i, \widehat{n}(i)-1)
$$

and

$$
\Gamma(\widehat{n}(i))>\Theta(i, \widehat{n}(i)) .
$$

From Proof of Proposition 3, we know that $\Gamma(n)$ is an increasing function of $n$ and, under some condition, $\Theta(i, n)$ decreases with respect to $n$. We can rewrite $\Theta(i, n)$ as follows:

$$
\Theta(i, n)=\frac{1}{n} \sum_{v=i}^{i+n-1} \prod_{z=v+1}^{i+n} \frac{1}{\lambda_{z}}
$$


$\Theta(i, n)$ is a function of $i$ such that:

(i) When $\lambda_{z}=\lambda, \forall z=2, \ldots, N$, then $\Theta(i, n)=\Theta\left(i^{\prime}, n\right) \forall i, i^{\prime}$.

(ii) When $\lambda_{z} \leq \lambda_{z+1}, \forall z=2, \ldots, N$, then $\Theta(i, n) \geq \Theta\left(i^{\prime}, n\right)$ for $i<i^{\prime}$.

(iii) When $\lambda_{z} \geq \lambda_{z+1}, \forall z=2, \ldots, N$, then $\Theta(i, n) \leq \Theta\left(i^{\prime}, n\right)$ for $i<i^{\prime}$.

Hence (i), (ii), (iii) and inequalities (26) and (27) lead to Proposition 5.

\subsection{Proof of Proposition 6.}

Let us consider the two following societies. In society $\mathbf{I}^{\prime}$, there are $N$ individuals characterized with $\sigma_{i}^{\prime 2}=1$. Hence, $\mathcal{P}^{\prime}=\left\{I^{\prime}\right\}$. In society $\mathbf{I}, n_{1}$ individuals are characterized with $\sigma_{1}^{2}$ and $n_{2}$ individuals are characterized with $\sigma_{2}^{2}$ such that $1>\sigma_{2}^{2}>\sigma_{1}^{2}$. Let us choose $\sigma_{1}^{2}$ and $\sigma_{2}^{2}$ such that $\mathcal{P}=\left\{S_{1}^{*}, S_{2}^{*}\right\}$ with $S_{1}^{*}$ (respectively $S_{2}^{*}$ ) comprised of the $n_{1}$ (respectively $n_{2}$ ) individuals with $\sigma_{1}^{2}$ (respectively $\left.\sigma_{2}^{2}\right)$. Hence, $\sigma_{1}^{2}, \sigma_{2}^{2}, n_{1}, n_{2}$ and $x$ are such that

$$
\frac{n_{1} \sigma_{1}^{2}+x \sigma_{2}^{2}}{\left(n_{1}+x\right)^{2}}>\frac{n_{1} \sigma_{1}^{2}}{\left(n_{1}\right)^{2}} \text { for all } x \in\left\{1, \ldots, n_{2}\right\}
$$

which is equivalent to

$$
\sigma_{2}^{2}>\sigma_{1}^{2} \frac{2 n_{1}+x}{n_{1}}
$$

As the RHS is an increasing function of $x$, a sufficient condition for this inequality to hold is

$$
\sigma_{2}^{2}>\sigma_{1}^{2} \frac{2 n_{1}+n_{2}}{n_{1}} .
$$

Thus, given both core partitions, we deduce that

$$
\bar{\pi}(\mathcal{P})=\frac{\alpha}{2} \frac{1}{N}\left(n_{1} \frac{n_{1} \sigma_{1}^{2}}{\left(n_{1}\right)^{2}}+n_{2} \frac{n_{2} \sigma_{2}^{2}}{\left(n_{2}\right)^{2}}\right)+\frac{\alpha}{2} \sigma_{\nu}^{2} \text { and } \bar{\pi}\left(\mathcal{P}^{\prime}\right)=\frac{\alpha}{2} \frac{1}{N}+\frac{\alpha}{2} \sigma_{\nu}^{2} .
$$

In order to have $\bar{\pi}(\mathcal{P})>\bar{\pi}\left(\mathcal{P}^{\prime}\right), \sigma_{1}^{2}$ and $\sigma_{2}^{2}$ must be such that:

$$
\sigma_{1}^{2}+\sigma_{2}^{2}>1
$$

Clearly there exist $\sigma_{1}^{2}$ and $\sigma_{2}^{2}$ that satisfy the following inequalities:

$$
1>\sigma_{1}^{2} ; 1>\sigma_{2}^{2} ; \sigma_{1}^{2}+\sigma_{2}^{2}>1 ; \sigma_{2}^{2}>\sigma_{1}^{2} \frac{2 n_{1}+n_{2}}{n_{1}} .
$$

For example, take $\sigma_{1}^{2}<\frac{n_{1}}{3 n_{1}+n_{2}}$ which satisfies $1>\sigma_{1}^{2}$. As $\sigma_{1}^{2}>0$, we have $1>1-\sigma_{1}^{2}$. Notice that $\sigma_{1}^{2}<\frac{n_{1}}{3 n_{1}+n_{2}}$ is equivalent to $1-\sigma_{1}^{2}>\sigma_{1}^{2} \frac{2 n_{1}+n_{2}}{n_{1}}$. So that for any $\sigma_{2}^{2}$ such that $1>\sigma_{2}^{2}>1-\sigma_{1}^{2}$, the four inequalities are satisfied. 


\subsection{Proof of Lemma 1.}

By contradiction, let us assume that two individuals $i$ and $j$ with $\sigma_{i}^{2}=\sigma_{j}^{2}=\sigma^{2}(z)$ are such that $i \in S$ and $j \notin S$. From (7), if $i \in S$, then

$$
\sigma_{i}^{2} \leq[2 n-1] \sum_{k \in S \backslash\{i\}} \frac{\sigma_{k}^{2}}{(n-1)^{2}}
$$

with $n$ being the size of $S$. But, from (8) if $j \notin S$, then

$$
\sigma_{j}^{2}>[2 n+1] \frac{\sigma_{i}^{2}+\sum_{k \in S_{j} \backslash\{i\}} \sigma_{k}^{2}}{n^{2}}
$$

As by assumption $\sigma_{i}^{2}=\sigma_{j}^{2}$, this inequality implies that

$$
\left(n^{2}-2 n-1\right) \sigma_{i}^{2}>[2 n+1] \sum_{k \in S_{j} \backslash\{i\}} \sigma_{k}^{2}
$$

When $n \leq 2, n^{2}-2 n-1<0$, then the LHS of (30) is negative while the RHS of (30) is positive. This leads to a contradiction. When $n>2, n^{2}-2 n-1>0$, equations (28) and (30) imply

$$
\frac{\left[2 n_{j}-1\right]}{\left(n_{j}-1\right)^{2}}>\frac{\left[2 n_{j}+1\right]}{n_{j}^{2}-2 n-1}
$$

leading to

$$
1>\frac{2 n_{j}-1}{2 n_{j}+1}>\frac{n_{j}^{2}-2 n+1}{n_{j}^{2}-2 n-1}>1
$$

which is a contradiction. Hence the result.

\subsection{Proof of Proposition 8.}

We look for a condition such that the introduction of $\widehat{i}$ modifies the set of pivotal agents. For simplicity, we focus on $S_{1}$ characterized by $\sigma_{p_{1}}^{2}$ satisfying (7) and assume that an individual characterized by $\sigma_{p_{1}}^{2}+\varepsilon, \varepsilon$ arbitrarily small, would not have been accepted in $S_{1}$

$$
\sigma_{p_{1}}^{2}+\varepsilon>\left(\frac{2 n_{j}+1}{n_{j}^{2}}\right)\left(\sum_{k \in S_{1} \backslash\left\{p_{1}\right\}} \sigma_{k}^{2}+\sigma_{p_{1}}^{2}\right)
$$

Let $L \equiv \sigma_{p_{1}}^{2}-\sigma_{p_{1}-1}^{2}>0$ and $\widehat{\sigma}^{2} \equiv \sigma_{p_{1}-1}^{2}+\delta, L>\delta>0$. Obviously, $\widehat{i}$ is accepted in $S_{1} \backslash\left\{p_{1}\right\}$.

Let us show that for given values of $L$ and $\delta$, we can find $\varepsilon$ such that $\widehat{i}$ is accepted in $S_{1} \backslash\left\{p_{1}\right\}$ and $p_{1}$ is rejected by $S_{1} \backslash\left\{p_{1}\right\} \cup\{\hat{i}\}$. Formally, the rejection of $p_{1}$ is written as

$$
\sigma_{p_{1}}^{2}>\left(\frac{2 n_{j}+1}{n_{j}^{2}}\right)\left(\sum_{k \in S_{1} \backslash\left\{p_{1}\right\}} \sigma_{k}^{2}+\widehat{\sigma}^{2}\right) .
$$


A sufficient condition for (32) given (31) writes

$$
\frac{2 n_{j}+1}{n_{j}^{2}}(L-\delta)>\varepsilon .
$$

As the LHS of the above inequality is strictly positive, it is always possible to find a distribution of risks with a value of $\varepsilon$ sufficiently small such that this inequality is satisfied.

\subsection{Proof of Lemma 2.}

Let us first consider that the grand coalition is formed and individuals allocate Pareto-optimally the grand coalition resources. Given (1), we have for any $i$ in $\mathbf{I}$ :

$$
c_{i}(\mathbf{I})=\frac{1}{\alpha_{i}}\left[\ln \mu_{i}(\mathbf{I})-\frac{\sum_{k \in \mathbf{I}} \frac{\ln \mu_{k}(\mathbf{I})}{\alpha_{k}}}{\sum_{k \in \mathbf{I}} \frac{1}{\alpha_{k}}}\right]+\frac{\frac{1}{\alpha_{i}}}{\sum_{k \in \mathbf{I}} \frac{1}{\alpha_{k}}} \sum_{k \in \mathbf{I}}\left(w_{k}+\varepsilon_{k}+\nu\right), \forall i \in \mathbf{I} .
$$

Given (33) the conditional expectation of individual consumption is used by econometricians when testing for the perfect risk sharing hypothesis:

$$
\mathbb{E}\left(c_{i t} \mid \frac{Y_{t}^{\mathbf{I}}\left(\epsilon_{t}^{\mathbf{I}}\right)}{N}, y_{i t}\right)=\kappa_{i}+\beta_{i} \frac{Y_{t}^{\mathbf{I}}}{N}+\zeta_{i} y_{i t}
$$

where the formulas of $\beta_{i}$ and $\zeta_{i}$ are obtained by using properties of conditional expectations of multivariate normal distributions (Ramanathan, 1993):

$$
\begin{aligned}
\beta_{i} & =\frac{\operatorname{cov}\left(\frac{Y_{t}^{\mathbf{I}}}{N}, c_{i t}\right) \operatorname{var}\left(y_{i t}\right)-\operatorname{cov}\left(y_{i t}, c_{i t}\right) \operatorname{cov}\left(\frac{Y_{t}^{\mathbf{I}}}{N}, y_{i t}\right)}{\operatorname{var}\left(\frac{Y_{t}^{\mathbf{I}}}{N}\right) \operatorname{var}\left(y_{i t}\right)-\left[\operatorname{cov}\left(\frac{Y_{t}^{\mathbf{I}}}{N}, y_{i t}\right)\right]^{2}} \\
\zeta_{i} & =\frac{\operatorname{cov}\left(y_{i t}, c_{i t}\right) \operatorname{var}\left(\frac{Y_{t}^{\mathbf{I}}}{N}\right)-\operatorname{cov}\left(\frac{Y_{t}^{\mathbf{I}}}{N}, c_{i t}\right) \operatorname{cov}\left(\frac{Y_{t}^{\mathbf{I}}}{N}, y_{i t}\right)}{\operatorname{var}\left(y_{i t}\right) \operatorname{var}\left(\frac{Y_{t}^{\mathbf{I}}}{N}\right)-\left[\operatorname{cov}\left(\frac{Y_{t}^{\mathbf{I}}}{N}, y_{i t}\right)\right]^{2}} .
\end{aligned}
$$

Given (33), some straightforward computations lead to the following

$$
\begin{aligned}
& \beta_{i}=\frac{\frac{1}{\alpha_{i}}}{\sum_{k \in \mathbf{I}} \frac{1}{\alpha_{k}}} \frac{\left(N \sigma_{\nu}^{2}+\frac{\sum_{m \in \mathbf{I}} \sigma_{m}^{2}}{N}\right)\left(\sigma_{\nu}^{2}+\sigma_{i}^{2}\right)-\left(N \sigma_{\nu}^{2}+\sigma_{i}^{2}\right)\left(\sigma_{\nu}^{2}+\frac{\sigma_{i}^{2}}{N}\right)}{\left(\sigma_{\nu}^{2}+\sigma_{i}^{2}\right)\left(\sigma_{\nu}^{2}+\frac{\sum_{m \in \mathbf{I}} \sigma_{m}^{2}}{N^{2}}\right)-\left(\sigma_{\nu}^{2}+\frac{\sigma_{i}^{2}}{N}\right)^{2}} \\
& \zeta_{i}=\frac{\frac{1}{\alpha_{i}}}{\sum_{k \in \mathbf{I}} \frac{1}{\alpha_{k}}} \frac{\left(N \sigma_{\nu}^{2}+\sigma_{i}^{2}\right)\left(\sigma_{\nu}^{2}+\frac{\sum_{m \in \mathbf{I}} \sigma_{m}^{2}}{N^{2}}\right)-\left(N \sigma_{\nu}^{2}+\frac{\sum_{m \in \mathbf{I}} \sigma_{m}^{2}}{N}\right)\left(\sigma_{\nu}^{2}+\frac{\sigma_{i}^{2}}{N}\right)}{\left(\sigma_{\nu}^{2}+\sigma_{i}^{2}\right)\left(\sigma_{\nu}^{2}+\frac{\sum_{m \in \mathbf{I}} \sigma_{m}^{2}}{N^{2}}\right)-\left(\sigma_{\nu}^{2}+\frac{\sigma_{i}^{2}}{N}\right)^{2}} .
\end{aligned}
$$

Hence,

$$
\beta_{i}=\frac{\frac{1}{\alpha_{i}}}{\sum_{k \in \mathbf{I}} \frac{1}{\alpha_{k}}} N
$$

and

$$
\zeta_{i}=0
$$


Second, let us now assume that optimal risk sharing takes place in subset $S \subset \mathbf{I}$, with $n \equiv$ $\operatorname{card}(S)<N$. It turns out that Pareto-optimal consumptions can now be written as follows

$$
c_{i t}=\frac{1}{\alpha_{i}}\left[\ln \mu_{i}-\frac{\sum_{k \in S} \frac{\ln \mu_{k}}{\alpha_{k}}}{\sum_{k \in S} \frac{1}{\alpha_{k}}}\right]+\frac{\frac{1}{\alpha_{i}} n}{\sum_{k \in S} \frac{1}{\alpha_{k}}} \frac{\sum_{k \in S}\left(w_{k t}+\varepsilon_{k t}+\nu_{t}\right)}{n}, \text { for } i \in S .
$$

If we still consider (34) and use the latter expression of $c_{i t}$ to compute (35a) and (35b), it turns out that coefficients $\beta_{i}$ and $\zeta_{i}$ are equal to:

$$
\begin{gathered}
\beta_{i}=\frac{\frac{1}{\alpha_{i}}}{\sum_{k \in S} \frac{1}{\alpha_{k}}} \frac{\left(n \sigma_{\nu}^{2}+\frac{\sum_{k \in S} \sigma_{k}^{2}}{N}\right)\left(\sigma_{\nu}^{2}+\sigma_{i}^{2}\right)-\left(n \sigma_{\nu}^{2}+\sigma_{i}^{2}\right)\left(\sigma_{\nu}^{2}+\frac{\sigma_{i}^{2}}{N}\right)}{\left(\sigma_{\nu}^{2}+\sigma_{i}^{2}\right)\left(\sigma_{\nu}^{2}+\frac{\sum_{m \in \mathbf{I}} \sigma_{m}^{2}}{N^{2}}\right)-\left(\sigma_{\nu}^{2}+\frac{\sigma_{i}^{2}}{N}\right)^{2}} \\
\zeta_{i}=\frac{\frac{1}{\alpha_{i}}}{\sum_{k \in S} \frac{1}{\alpha_{k}}} \frac{\left(n \sigma_{\nu}^{2}+\sigma_{i}^{2}\right)\left(\sigma_{\nu}^{2}+\frac{\sum_{m \in \mathbf{I}} \sigma_{m}^{2}}{N^{2}}\right)-\left(n \sigma_{\nu}^{2}+\frac{\sum_{k \in S} \sigma_{k}^{2}}{N}\right)\left(\sigma_{\nu}^{2}+\frac{\sigma_{i}^{2}}{N}\right)}{\left(\sigma_{\nu}^{2}+\sigma_{i}^{2}\right)\left(\sigma_{\nu}^{2}+\frac{\sum_{m \in \mathbf{I}} \sigma_{m}^{2}}{N^{2}}\right)-\left(\sigma_{\nu}^{2}+\frac{\sigma_{i}^{2}}{N}\right)^{2}}
\end{gathered}
$$

Dividing by $\left(\sigma_{i}^{2}\right)^{2}$ both the numerator and denominator in (37) and (38) leads to:

$$
\begin{gathered}
\beta_{i}=\frac{\frac{1}{\alpha_{i}}}{\sum_{k \in S} \frac{1}{\alpha_{k}}} \frac{\left(n \frac{\sigma_{\nu}^{2}}{\sigma_{i}^{2}}+\frac{\sum_{k \in S} \sigma_{k}^{2}}{\sigma_{i}^{2} N}\right)\left(\frac{\sigma_{\nu}^{2}}{\sigma_{i}^{2}}+1\right)-\left(n \frac{\sigma_{\nu}^{2}}{\sigma_{i}^{2}}+1\right)\left(\frac{\sigma_{\nu}^{2}}{\sigma_{i}^{2}}+\frac{1}{N}\right)}{\left(\frac{\sigma_{\nu}^{2}}{\sigma_{i}^{2}}+1\right)\left(\frac{\sigma_{\nu}^{2}}{\sigma_{i}^{2}}+\frac{\sum_{m \in \mathbf{I}} \sigma_{m}^{2}}{\sigma_{i}^{2} N^{2}}\right)-\left(\frac{\sigma_{\nu}^{2}}{\sigma_{i}^{2}}+\frac{1}{N}\right)^{2}} \\
\zeta_{i}=\frac{\frac{1}{\alpha_{i}}}{\sum_{k \in S} \frac{1}{\alpha_{k}}} \frac{\left(n \frac{\sigma_{\nu}^{2}}{\sigma_{i}^{2}}+1\right)\left(\frac{\sigma_{\nu}^{2}}{\sigma_{i}^{2}}+\frac{\sum_{m \in \mathbf{I}} \sigma_{m}^{2}}{\sigma_{i}^{2} N^{2}}\right)-\left(n \frac{\sigma_{\nu}^{2}}{\sigma_{i}^{2}}+\frac{\sum_{k \in S_{j}} \sigma_{k}^{2}}{\sigma_{i}^{2} N}\right)\left(\frac{\sigma_{\nu}^{2}}{\sigma_{i}^{2}}+\frac{1}{N}\right)}{\left(\frac{\sigma_{\nu}^{2}}{\sigma_{i}^{2}}+1\right)\left(\frac{\sigma_{\nu}^{2}}{\sigma_{i}^{2}}+\frac{\sum_{m \in \mathbf{I}} \sigma_{m}^{2}}{\sigma_{i}^{2} N^{2}}\right)-\left(\frac{\sigma_{\nu}^{2}}{\sigma_{i}^{2}}+\frac{1}{N}\right)^{2}} .
\end{gathered}
$$

If we assume that $\lim _{N \rightarrow \infty} \frac{\sigma_{N}^{2}}{\sigma_{1}^{2}}<\infty$, this implies that

$$
\lim _{N \rightarrow \infty} \frac{\sigma_{N}^{2}}{N \sigma_{1}^{2}}=0
$$

Further, as $\frac{\sum_{m \in \mathbf{I}} \sigma_{m}^{2}}{N^{2} \sigma_{i}^{2}} \leq \frac{\sigma_{N}^{2}}{\sigma_{1}^{2} N} \forall i=1, \ldots, N$, we thus easily deduce that when $\lim _{N \rightarrow \infty} \frac{\sigma_{N}^{2}}{\sigma_{1}^{2}}<\infty$, then

$$
\lim _{N \rightarrow \infty} \frac{\sum_{m \in \mathbf{I}} \sigma_{m}^{2}}{N^{2} \sigma_{i}^{2}}=0, \forall i=1, \ldots, N
$$

If $N$ tends to infinity, the following equalities obtain:

$$
\begin{aligned}
\beta_{i} & \simeq \frac{\frac{1}{\alpha_{i}}}{\sum_{k \in S} \frac{1}{\alpha_{k}}} \frac{\left(n \frac{\sigma_{\nu}^{2}}{\sigma_{i}^{2}}+\frac{\sum_{k \in S} \sigma_{k}^{2}}{\sigma_{i}^{2} N}\right)\left(\frac{\sigma_{\nu}^{2}}{\sigma_{i}^{2}}+1\right)-\left(n \frac{\sigma_{\nu}^{2}}{\sigma_{i}^{2}}+1\right)\left(\frac{\sigma_{\nu}^{2}}{\sigma_{i}^{2}}\right)}{\left(\frac{\sigma_{\nu}^{2}}{\sigma_{i}^{2}}+1\right)\left(\frac{\sigma_{\nu}^{2}}{\sigma_{i}^{2}}\right)-\left(\frac{\sigma_{\nu}^{2}}{\sigma_{i}^{2}}\right)^{2}} \\
\zeta_{i} & \simeq \frac{\frac{1}{\alpha_{i}}}{\sum_{k \in S} \frac{1}{\alpha_{k}}} \frac{\left(n \frac{\sigma_{\nu}^{2}}{\sigma_{i}^{2}}+1\right)\left(\frac{\sigma_{\nu}^{2}}{\sigma_{i}^{2}}\right)-\left(n \frac{\sigma_{\nu}^{2}}{\sigma_{i}^{2}}+\frac{\sum_{k \in S} \sigma_{k}^{2}}{\sigma_{i}^{2} N}\right)\left(\frac{\sigma_{\nu}^{2}}{\sigma_{i}^{2}}\right)}{\left(\frac{\sigma_{\nu}^{2}}{\sigma_{i}^{2}}+1\right)\left(\frac{\sigma_{\nu}^{2}}{\sigma_{i}^{2}}\right)-\left(\frac{\sigma_{\nu}^{2}}{\sigma_{i}^{2}}\right)^{2}} .
\end{aligned}
$$


As $\lim _{N \rightarrow \infty} \frac{\sigma_{N}^{2}}{\sigma_{1}^{2}}<\infty$ implying that $\lim _{N \rightarrow \infty} \frac{\sum_{m \in \mathbf{I}} \sigma_{m}^{2}}{N^{2} \sigma_{i}^{2}}=0$ and $\lim _{N \rightarrow \infty} \frac{\sum_{k \in S} \sigma_{k}^{2}}{\sigma_{i}^{2} N n}=0 \forall i=1, \ldots, N$, we get:

$$
\begin{gathered}
\beta_{i} \simeq \frac{\frac{1}{\alpha_{i}}}{\sum_{k \in S} \frac{1}{\alpha_{k}}}(n-1) \\
\zeta_{i} \simeq \frac{\frac{1}{\alpha_{i}}}{\sum_{k \in S} \frac{1}{\alpha_{k}}} .
\end{gathered}
$$

If we assume that each individual belongs to one risk-sharing coalition only, and society $\mathbf{I}$ is organized into $J$ risk-sharing coalitions, denoting by $\bar{\beta}_{\mathbf{I}} \equiv \frac{\sum_{i \in \mathbf{I}} \beta_{i}}{N}$ and $\bar{\zeta}_{\mathbf{I}} \equiv \frac{\sum_{i \in \mathbf{I}} \zeta_{i}}{N}$ we immediately get:

$$
\lim _{N \rightarrow+\infty} \bar{\beta}_{\mathbf{I}}=1-\frac{1}{\bar{n}_{J}}
$$

and

$$
\lim _{N \rightarrow+\infty} \bar{\zeta}_{\mathbf{I}}=\frac{1}{\bar{n}_{J}}
$$

with $\bar{n}_{J} \equiv \frac{N}{J}$.

\subsection{Proof of Proposition 9.}

We will denote by $S^{c}(i)$ the consecutive club whose lowest risky agent is individual $i$. Let us denote by $\widehat{n}(i \mid \Lambda)$ the size of $S^{c}(i)$ such that $\widehat{n}(i \mid \Lambda)=\arg \max V_{i}\left(S^{c}(i)\right)$, for a risk ratio schedule $\Lambda$.

We first offer the following Lemma

Lemma 3 For two societies $\mathbf{I}$ and $\mathbf{I}^{\prime}$ characterized respectively by $\Lambda=\left\{\lambda_{2}, \lambda_{3}, \ldots, \lambda_{N}\right\}$ and $\Lambda^{\prime}=$ $\left\{\lambda_{2}^{\prime}, \lambda_{3}^{\prime}, \ldots, \lambda_{N}^{\prime}\right\}$ with $\lambda_{z}<\lambda_{z}^{\prime}$ for $z=2, \ldots, N$, we have $\widehat{n}(i \mid \Lambda) \geq \widehat{n}\left(i \mid \Lambda^{\prime}\right)$.

Proof. Let us denote by $\Theta\left(\vec{\lambda}_{i, n}\right) \equiv \Theta(i, n)=\frac{1}{n} \sum_{v=i}^{i+n-1} \prod_{z=v+1}^{i+n} \frac{1}{\lambda_{z}}$ with $\vec{\lambda}_{i, n}=\left(\lambda_{i+1}, \lambda_{i+2}, \ldots, \lambda_{i+n-1}\right)$. Hence for two vectors $\vec{\lambda}_{i, n}$ and ${\overrightarrow{\lambda^{\prime}}}_{i, n}$ where $\lambda_{z}^{\prime}>\lambda_{z}, \forall z=i+1, \ldots, i+n-1$, we have $\Theta\left(\vec{\lambda}_{i, n}\right)>$ $\Theta\left({\overrightarrow{\lambda^{\prime}}}_{i, n}\right), \forall i \in \mathbf{I}$ and $\forall n=1, \ldots, N-i+1$. Given inequalities $(26)$ and (27) and that $\Theta\left(\vec{\lambda}_{i, n}\right)>$ $\Theta\left({\overrightarrow{\lambda^{\prime}}}_{i, n}\right)$, it is thus easy to deduce that the optimal size of the consecutive group beginning with agent $i$ is larger under $\Lambda=\left\{\lambda_{2}, \lambda_{3}, \ldots, \lambda_{N}\right\}$ than under $\Lambda^{\prime}=\left\{\lambda_{2}^{\prime}, \lambda_{3}^{\prime}, \ldots, \lambda_{N}^{\prime}\right\}$. Hence, Lemma 1 .

Lemma 4 Let us denote by $p_{S^{c}(i)}$ the pivotal agent of any consecutive club $S^{c}(i)$. For any society $\mathbf{I}$, any $i^{\prime}<i$ we have $p_{S^{c}(i)}>p_{S^{c}\left(i^{\prime}\right)}$.

Proof. We know that $\sigma_{p_{S^{c}(i)}}^{2}$ satisfies

$$
\sigma_{p_{S^{c}(i)}}^{2} \leq\left[2 n_{j}^{c}-1\right] \sum_{k=i}^{p_{S^{c}(i)}-1} \frac{\sigma_{k}^{2}}{\left(n_{j}^{c}-1\right)^{2}}
$$


and

$$
\sigma_{p_{S^{c}(i)}+1}^{2}>\left[2 n_{j}^{c}+1\right] \sum_{k=i}^{p_{S^{c}(i)}} \frac{\sigma_{k}^{2}}{n_{j}^{c 2}} .
$$

Let us consider the consecutive $\operatorname{club} S^{c}\left(i^{\prime}\right)=\left\{i^{\prime}, \ldots, p_{S^{c}(i)}+1\right\}$. By assumption on the individuals ordering, we have

$$
\sum_{k=i}^{p_{S^{c}(i)}} \frac{\sigma_{k}^{2}}{n_{j}^{c}}>\sum_{k=i^{\prime}}^{p_{S^{c}(i)}} \frac{\sigma_{k}^{2}}{n_{j}^{\prime c}} \text { for any } i^{\prime}<i .
$$

Hence as $\frac{2 n^{\prime}+1}{n^{\prime}}<\frac{2 n+1}{n}$ for any $n^{\prime}>n$, we thus have

$$
\sigma_{p_{S^{c}(i)}+1}^{2}>\left[2 n_{j}^{c}+1\right] \sum_{k=i}^{p_{S^{c}(i)}} \frac{\sigma_{k}^{2}}{n_{j}^{c 2}}>\left[2 n_{j}^{c}+1\right] \sum_{k=i^{\prime}}^{p_{S^{c}(i)}} \frac{\sigma_{k}^{2}}{n_{j}^{c 2}} \text {, for any } i^{\prime}<i .
$$

We easily deduce that $p_{S^{c}(i)}>p_{S^{c}\left(i^{\prime}\right)}$ for any $i^{\prime}<i$.

Let us now define $p_{j}^{*}(\Lambda)$ the pivotal agent of club $S_{j}$ in the core partition associated to $\Lambda$. Let us consider individual 1. Using Lemma 3 , for $\Lambda=\left\{\lambda_{2}, \lambda_{3}, \ldots, \lambda_{N}\right\}$ and $\Lambda^{\prime}=\left\{\lambda_{2}^{\prime}, \lambda_{3}^{\prime}, \ldots, \lambda_{N}^{\prime}\right\}$ with $\lambda_{z}<\lambda_{z}^{\prime}$ for $z=2, \ldots, N$, we deduce that $p_{1}^{*}(\Lambda) \geq p_{1}^{*}\left(\Lambda^{\prime}\right)$. Using Lemma 4 , we thus deduce that $p_{2}^{*}(\Lambda) \equiv p_{S^{c}\left(p_{1}(\Lambda)+1\right)}^{*}(\Lambda)>p_{S^{c}\left(p_{1}\left(\Lambda^{\prime}\right)+1\right)}(\Lambda)$. Using again Lemma 3 allows us to say that $p_{S^{c}\left(p_{1}\left(\Lambda^{\prime}\right)+1\right)}(\Lambda) \geq p_{S^{c}\left(p_{1}\left(\Lambda^{\prime}\right)+1\right)}^{*}\left(\Lambda^{\prime}\right) \equiv p_{2}^{*}\left(\Lambda^{\prime}\right)$. Hence $p_{2}^{*}(\Lambda) \geq p_{2}^{*}\left(\Lambda^{\prime}\right)$. Iterating this reasoning until $j=J$ allows us to say that $p_{j}^{*}(\Lambda) \geq p_{j}^{*}\left(\Lambda^{\prime}\right)$ for any $j=1, \ldots, J$. Hence for any $i=1, \ldots, N$ we thus deduce that the number of pivotal agents associated with $\Lambda$ such that $p_{j}^{*}(\Lambda) \leq i$ compared to the number of pivotal agents associated with $\Lambda^{\prime} \operatorname{such} p_{j}\left(\Lambda^{\prime}\right) \leq i$ is higher for $\Lambda$ than $\Lambda^{\prime}$. This ends proof of Proposition 9.

\subsection{Proof of Proposition 10.}

Existence. It is easy to see that in our case the common ranking property is also satisfied. Hence, a core partition exists.

Proof of (ii): Consecutivity. By contradiction, let us consider a core-partition $\mathcal{P}^{*}$ characterized by some non consecutive groups, that is, there exist individual $i, \widetilde{i} \in S_{j}^{*}$ and $i^{\prime} \in S_{j^{\prime}}^{*}$ with $i<i^{\prime}<\widetilde{i}$.

Suppose first that $\pi^{z}\left(S_{j}^{*}\right) \geq \pi^{z}\left(S_{j^{\prime}}^{*}\right)$ for any $z=1, \ldots, N$. As $i<i^{\prime}<\widetilde{i} \Longleftrightarrow \frac{1}{\alpha_{i}}>\frac{1}{\alpha_{i^{\prime}}}>\frac{1}{\alpha_{\tilde{i}}}$, we have

$$
\forall z \in\left(S_{j^{\prime}}^{*} \backslash\left\{i^{\prime}\right\}\right) \cup\{i\}, V_{z}\left(\left(S_{j^{\prime}}^{*} \backslash\left\{i^{\prime}\right\}\right) \cup\{i\}\right)>V_{z}\left(\mathcal{P}^{*}\right) .
$$

Second, assume that $\pi^{z}\left(S_{j^{\prime}}^{*}\right) \geq \pi^{z}\left(S_{j}^{*}\right)$ for any $z=1, \ldots, N$. We have

$$
\forall z \in\left(S_{j}^{*} \backslash\{\widetilde{i}\}\right) \cup\left\{i^{\prime}\right\}, V_{z}\left(\left(S_{j}^{*} \backslash\{\widetilde{i}\}\right) \cup\left\{i^{\prime}\right\}\right)>V_{z}\left(\mathcal{P}^{*}\right) .
$$

Hence a contradiction with the fact that $\mathcal{P}^{*}$ is assumed to be a core-partition. 


\section{Proof of (i): Uniqueness.}

Knowing that core partition satisfies the consecutivity property, an individual $z$ is accepted if and only if

$$
-\frac{n_{j}^{2}}{\left(\sum_{k \in S} \frac{1}{\alpha_{k}}\right)^{2}}\left(\sigma_{\nu}^{2}+\frac{\sigma_{\varepsilon}^{2}}{n_{j}}\right) \geq-\frac{\left(n_{j}+1\right)^{2}}{\left(\sum_{k \in S} \frac{1}{\alpha_{k}}+\frac{1}{\alpha_{z}}\right)^{2}}\left(\sigma_{\nu}^{2}+\frac{\sigma_{\varepsilon}^{2}}{\left(n_{j}+1\right)}\right)
$$

which amounts to

$$
-\left(\frac{1}{\alpha_{z}}\right)^{2} n_{j}^{2}\left(\sigma_{\nu}^{2}+\frac{\sigma_{\varepsilon}^{2}}{n_{j}}\right)-\left(\frac{1}{\alpha_{z}}\right) 2 n_{j}^{2}\left(\sum_{k \in S} \frac{1}{\alpha_{k}}\right)\left(\sigma_{\nu}^{2}+\frac{\sigma_{\varepsilon}^{2}}{n_{j}}\right)+\left(\sum_{k \in S} \frac{1}{\alpha_{k}}\right)^{2}\left(\left(2 n_{j}+1\right) \sigma_{\nu}^{2}+\sigma_{\varepsilon}^{2}\right) \geq 0 .
$$

For positive $\alpha_{z}$, the LHS of the above inequality is positive if and only if

$$
\frac{\sum_{k \in S_{j}} \frac{1}{\alpha_{k}}}{\frac{1}{\alpha_{z}} n_{j}}\left(-n_{j}+\sqrt{\left(n_{j}+1\right)^{2} \frac{\left(\sigma_{\nu}^{2}+\frac{\sigma_{\varepsilon}^{2}}{\left(n_{j}+1\right)}\right)}{\left(\sigma_{\nu}^{2}+\frac{\sigma_{\varepsilon}^{2}}{n_{j}}\right)}}\right) \leq 1 .
$$

The aim is to show that the LHS of this inequality is monotonously increasing with the size of the coalition.

First, the expression $\left(-n_{j}+\sqrt{\left(n_{j}+1\right)^{2} \frac{\left(\sigma_{\nu}^{2}+\frac{\sigma_{\varepsilon}^{2}}{\left(n_{j}+1\right)}\right)}{\left(\sigma_{\nu}^{2}+\frac{\sigma_{\varepsilon}^{2}}{n_{j}}\right)}}\right)$ is increasing with respect to $n$ (we omit $j$ for convenience) if and only if:

$$
\begin{aligned}
& 2\left[n(n+1)+\frac{\sigma_{\varepsilon}^{2}}{\sigma_{\nu}^{2}} n\right]\left[n+\frac{\sigma_{\varepsilon}^{2}}{\sigma_{\nu}^{2}}\right]+\left[2 n+1+\frac{\sigma_{\varepsilon}^{2}}{\sigma_{\nu}^{2}}\right] \frac{\sigma_{\varepsilon}^{2}}{\sigma_{\nu}^{2}} \\
& >2\left[n+\frac{\sigma_{\varepsilon}^{2}}{\sigma_{\nu}^{2}}\right] \sqrt{\left[n(n+1)+\frac{\sigma_{\varepsilon}^{2}}{\sigma_{\nu}^{2}} n\right]\left[n(n+1)+\frac{\sigma_{\varepsilon}^{2}}{\sigma_{\nu}^{2}}(n+1)\right]} .
\end{aligned}
$$

Using the fact that

$$
\left[n(n+1)+\frac{\sigma_{\varepsilon}^{2}}{\sigma_{\nu}^{2}}\left(n+\frac{1}{2}\right)\right]^{2}>\left[n(n+1)+\frac{\sigma_{\varepsilon}^{2}}{\sigma_{\nu}^{2}} n\right]\left[n(n+1)+\frac{\sigma_{\varepsilon}^{2}}{\sigma_{\nu}^{2}}(n+1)\right]
$$

we can show that inequality (49) is satisfied whatever $\frac{\sigma_{\varepsilon}^{2}}{\sigma_{\nu}^{2}}$ and $n$.

Second, we offer a sufficient condition such that the ratio $\frac{\sum_{k \in S_{j}} \frac{1}{\alpha_{k}}}{\frac{1}{\alpha_{z}} n_{j}}$ increases with respect to the size of the coalition $S_{j}$, i.e.

$$
\frac{\sum_{k \in S_{j}} \frac{1}{\alpha_{k}}+\frac{1}{\alpha_{z}}}{\frac{1}{\alpha_{z+1}}\left(n_{j}+1\right)}-\frac{\sum_{k \in S_{j}} \frac{1}{\alpha_{k}}}{\frac{1}{\alpha_{z}} n_{j}} \geq 0
$$

which is equivalent to

$$
\left(n_{j}-\frac{\frac{1}{\alpha_{z+1}}}{\frac{1}{\alpha_{z}}}\left(n_{j}+1\right)\right)\left(\sum_{k \in S_{j}} \frac{1}{\alpha_{k}}\right)+n_{j} \frac{1}{\alpha_{z}} \geq 0
$$



$\forall n \geq 1$.

Let us define $\Phi(i, n)=\left(n-\frac{\frac{1}{\alpha_{i+n+1}}}{\frac{1}{\alpha_{i+n}}}(n+1)\right)\left(\sum_{k=i}^{i+n-1} \frac{1}{\alpha_{k}}\right)+n \frac{1}{\alpha_{i+n}}$ and show that $\Phi(i, n) \geq 0$,

We have $\Phi(i, 1)=\left(1-\frac{\frac{1}{\alpha_{i+2}}}{\frac{1}{\alpha_{i+1}}}(2)\right)\left(\frac{1}{\alpha_{i}}\right)+\frac{1}{\alpha_{i+1}}$. With $\chi_{i}=\frac{1 / \alpha_{i}}{1 / \alpha_{i-1}}<1$ whatever $i, \Phi(i, 1)$ is positive if and only if

$$
1 \geq \frac{\chi_{i+2}-\chi_{i+1}}{1-\chi_{i+2}}, \text { whatever } i=1, \ldots, N .
$$

Let us show that $\Phi(i, n)$ is monotonously increasing with respect to $n$, that is,

$$
\begin{aligned}
\Phi(i, n+1)-\Phi(i, n) & =\left(n+1-\frac{\frac{1}{\alpha_{i+n+2}}}{\frac{1}{\alpha_{i+n+1}}}(n+2)\right)\left(\sum_{k=i}^{i+n-1} \frac{1}{\alpha_{k}}+\frac{1}{\alpha_{i+n}}\right) \\
& +(n+1) \frac{1}{\alpha_{i+n+1}}-\left(n-\frac{\frac{1}{\alpha_{i+n+1}}}{\frac{1}{\alpha_{i+n}}}(n+1)\right)\left(\sum_{k=i}^{i+n-1} \frac{1}{\alpha_{k}}\right)-n \frac{1}{\alpha_{i+n}}
\end{aligned}
$$

which is equivalent to

$$
\begin{aligned}
\Phi(i, n+1)-\Phi(i, n) & =\left(\sum_{k=i}^{i+n-1} \frac{1}{\alpha_{k}}\right)\left(1-\frac{\frac{1}{\alpha_{i+n+2}}}{\frac{1}{\alpha_{i+n+1}}}(n+2)+\frac{\frac{1}{\alpha_{i+n+1}}}{\frac{1}{\alpha_{i+n}}}(n+1)\right) \\
& +\left(1-\frac{\overline{\alpha_{i+n+2}}}{\frac{1}{\alpha_{i+n+1}}}(n+2)\right) \frac{1}{\alpha_{i+n}}+(n+1) \frac{1}{\alpha_{i+n+1}} \\
& \Leftrightarrow \\
\Phi(i, n+1)-\Phi(i, n) & =\left(\sum_{k=i}^{i+n-1} \frac{1}{\alpha_{k}}\right)\left(1-\chi_{i+n+2}(n+2)+\chi_{i+n+1}(n+1)\right) \\
& +\left(1-\lambda_{i+n+2}(n+2)\right) \frac{1}{\alpha_{i+n}}+(n+1) \frac{1}{\alpha_{i+n+1}} .
\end{aligned}
$$

With $\chi_{i+n+1} \equiv \frac{\alpha_{i+n}}{\alpha_{i+n+1}}$, we have

$$
\Phi(i, n+1)-\Phi(i, n)=\left(\left(\sum_{k=i}^{i+n-1} \frac{1}{\alpha_{k}}\right)+\frac{1}{\alpha_{i+n}}\right)\left(1-\chi_{i+n+2}(n+2)+\chi_{i+n+1}(n+1)\right) .
$$

Hence,

$$
\Phi(i, n+1)-\Phi(i, n) \geq 0 \Leftrightarrow 1 \geq(n+1) \frac{\left(\chi_{i+n+2}-\chi_{i+n+1}\right)}{\left(1-\chi_{i+n+2}\right)} .
$$

With $z \equiv i+n+1$, we can rewrite this inequality as follows

$$
1 \geq\left(\frac{z-i}{z+1}\right)(z+1) \frac{\left(\chi_{z+1}-\chi_{z}\right)}{\left(1-\chi_{z+1}\right)}
$$

As $0 \leq \frac{(z-i)}{(z+1)} \leq 1$, we deduce that if for all $z=3, \ldots, N-1, \frac{\chi_{z}-\chi_{z+1}}{\chi_{z+1}-1}(z+1) \leq 1$, then $\triangle \Phi(i, n) \geq 0$. We deduce that if for all $z=2, \ldots, N-1, \frac{\chi_{z}-\chi_{z+1}}{\chi_{z+1}-1}(z+1) \leq 1$ then $\triangle \Phi(i, n) \geq 0$ and $\Phi(i, 1) \geq 0$, $\forall i=1, \ldots, N$.

Proof of (iii): The proof is identical to Proof of (iii) of Proposition 5 except with the fact that the risk premium $\pi\left(S_{j}\right)$ must now be replaced by $\pi^{z}\left(S_{j}\right)$. 


\subsection{Proposition 11.}

Let us define $\widetilde{\Gamma}(n)=\left(-n+{\sqrt{(n+1)^{2} \frac{\left(\sigma_{\nu}^{2}+\frac{\sigma_{\varepsilon}^{2}}{n+1}\right)}{\left(\sigma_{\nu}^{2}+\frac{\sigma_{\varepsilon}^{2}}{n}\right)}}}^{-1}\right.$ and $\widetilde{\Theta}(i, n)=\frac{1}{n} \frac{\sum_{k=i}^{i+n-1} \frac{1}{\alpha_{k}}}{\frac{1}{\alpha_{i+n}}}$. We will denote by $\widehat{n}(i)$ the size of $S^{c}(i)$ such that $\widehat{n}(i)=\arg \max V_{i}\left(S^{c}(i)\right)$ in the subset $\mathbf{I} \backslash\{1,2, \ldots, i-1\}$, for a risk ratio schedule $\Lambda$. Hence, $\widehat{n}(i)$ satisfies inequalities characterizing a pivotal agent:

$$
\widetilde{\Gamma}(\widehat{n}(i)-1) \geq \widetilde{\Theta}(i, \widehat{n}(i)-1)
$$

and

$$
\widetilde{\Gamma}(\widehat{n}(i))<\widetilde{\Theta}(i, \widehat{n}(i)) .
$$

From Proof of Proposition 10, we deduce that $\widetilde{\Gamma}(n)$ is a decreasing function of $n$ and, under some condition, $\Theta(i, n)$ increases with respect to $n$. We can rewrite $\Theta(i, n)$ as follows: $\frac{1}{n} \frac{\sum_{k=i}^{i+n-1} \frac{1}{\alpha_{k}}}{\frac{1}{\alpha_{i+n}}}$

$$
\Theta(i, n)=\frac{1}{n} \sum_{v=i}^{i+n-1} \prod_{z=v+1}^{i+n} \frac{1}{\chi_{z}}
$$

$\Theta(i, n)$ is a function of $i$ such that:

(i) When $\chi_{z}=\chi, \forall z=2, \ldots, N$, then $\Theta(i, n)=\Theta\left(i^{\prime}, n\right) \forall i, i^{\prime}$.

(ii) When $\chi_{z} \leq \chi_{z+1}, \forall z=2, \ldots, N$, then $\Theta(i, n) \geq \Theta\left(i^{\prime}, n\right)$ for $i<i^{\prime}$.

(iii) When $\chi_{z} \geq \chi_{z+1}, \forall z=2, \ldots, N$, then $\Theta(i, n) \leq \Theta\left(i^{\prime}, n\right)$ for $i<i^{\prime}$.

Hence, (i), (ii), (iii) and inequalities (50) and (51) lead to Proposition 11. 